\title{
ProbFlow: Joint Optical Flow and Uncertainty Estimation
}

\author{
Anne S. Wannenwetsch ${ }^{1}$ \\ ${ }^{1}$ TU Darmstadt
}

\author{
Margret Keuper ${ }^{2} \quad$ Stefan Roth $^{1}$ \\ ${ }^{2}$ University of Mannheim
}

\begin{abstract}
Optical flow estimation remains challenging due to untextured areas, motion boundaries, occlusions, and more. Thus, the estimated flow is not equally reliable across the image. To that end, post-hoc confidence measures have been introduced to assess the per-pixel reliability of the flow. We overcome the artificial separation of optical flow and confidence estimation by introducing a method that jointly predicts optical flow and its underlying uncertainty. Starting from common energy-based formulations, we rely on the corresponding posterior distribution of the flow given the images. We derive a variational inference scheme based on mean field, which incorporates best practices from energy minimization. An uncertainty measure is obtained along the flow at every pixel as the (marginal) entropy of the variational distribution. We demonstrate the flexibility of our probabilistic approach by applying it to two different energies and on two benchmarks. We not only obtain flow results that are competitive with the underlying energy minimization approach, but also a reliable uncertainty measure that significantly outperforms existing post-hoc approaches.
\end{abstract}

\section{Introduction}

Optical flow estimation has been extensively studied for more than three decades $5,9,14,21,36$. However, motion boundaries, large displacements, and occlusions still lead to erroneous flow fields, especially in challenging imaging conditions. In contrast, flow predictions are almost errorless in textured regions of uniform motion, which causes the reliability of optical flow predictions to vary greatly across the image. Uncertainty measure $\$$ aim to predict the reliability of the flow estimates and rate each estimate according to its predicted accuracy $24,27,30$. One application of such measures is to improve optical flow estimation using different ways of post-processing 4, 36. Moreover, uncertainty measures represent an important tool when optical flow is used as a cue for other computer vision tasks, such

\footnotetext{
${ }^{1}$ Uncertainty measures are closely related to confidence measures as their values are inversely related to confidence estimates.
}

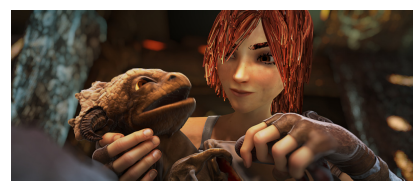

(a) First frame of image pair

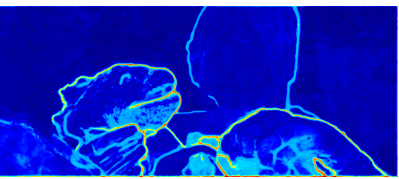

(c) Uncertainty map from our method

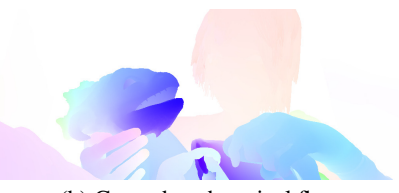

(b) Ground truth optical flow

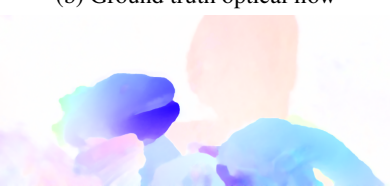

(d) Flow estimate from our method
Figure 1 . Our uncertainty measure accurately detects regions with reliable flow predictions (dark blue) as well as parts of the image with erroneous estimates (dark red). Best viewed on screen.

as image segmentation or tracking, which consequently depend on the correctness of the flow. In such cases, error propagation can be avoided if only flow estimates of high confidence are taken into consideration, e.g. 33,47.

Several post-hoc confidence measures have been introduced to directly estimate the uncertainty of flow predictions, e.g. 3, 20, 24, 30]. However, optical flow estimation is known to be rather challenging as a one-shot process 14, 15,43. We thus believe it is natural to ask why confidence estimation should be restricted to non-iterative (posthoc) approaches. Moreover, flow algorithms for a concrete problem are carefully selected for the task at hand and provide us with an underlying model. We, thus, argue that it is very desirable to apply a model-inherent uncertainty measure tailored to the chosen method. We even go one step further and aim to jointly predict optical flow and a corresponding uncertainty in order to preserve and extract important information contained in the flow estimation process.

Our work focuses on energy-based methods $3,15,43$ as they represent a flexible framework in widespread use. Starting from a general energy formulation, we derive a probability distribution of the flow field conditioned on the input images. In this setting, optical flow can be determined by minimizing the expected loss under the modeled posterior and the corresponding uncertainty measure is naturally obtained as the marginal entropy of the posterior at every pixel. Due to the intractability of exact posterior inference in general, we rely on variational approximate inference and 
use a mean-field approach. In this way, flow predictions and uncertainty estimations can be obtained as the result of a joint minimization problem that makes an additional training step of the uncertainty measure unnecessary 17,30 . Fig. 1 shows an exemplary flow field and uncertainty prediction of our probabilistic approach obtained on the Sintel benchmark [11. We term our approach ProbFlow.

To the best of our knowledge, we introduce the first fully probabilistic flow estimation approach that shows competitive results on established benchmarks. Moreover, our work is unique in that it is broadly applicable to a large number of existing energy-based optical flow algorithms and that we demonstrate how to benefit from the probabilistic framework in the context of uncertainty measures.

We apply ProbFlow to a classic Horn-Schunck-style energy as proposed in 43 and to the more recent EpicFlow formulation 36]. We show that the obtained flow fields are competitive with or even outperform the corresponding energy minimization results on the Middlebury 22 and Sintel [11] datasets. To assess the performance of the uncertainties estimated by our method, we rely on existing evaluation approaches and propose a new criterion based on the Spearman's rank correlation coefficient. Our uncertainty measure is clearly superior in comparison to various competing approaches and significantly outperforms the best existing methods. We further show the benefits of our uncertainty estimates in an application to motion segmentation 22 by generating highly reliable point trajectories.

\section{Related Work}

Starting from the work of Horn and Schunck [21, global energy minimization approaches have been used extensively for optical flow estimation; see [3, 15, 43 for indepth overviews of existing methods and best practices. Despite their conceptual simplicity, global energy formulations are still up-to-date [13,29]. Moreover, energy-based approaches are frequently used for post-processing optical flow predictions obtained through other means $[1,14,36$.

In the past, a variety of optical flow uncertainty measures have been proposed. Various simple methods are based on the characteristics of the input data only, e.g. 3, 20]. We refer to 24.27 for a more comprehensive summary of such confidence approaches. In comparison to our work, all of these measures omit important sources of information by not considering the estimated optical flow field itself.

A second class of confidence measures relies on an analysis of the estimated flow fields. Kondermann et al. 23 learn a linear subspace of true spatio-temporal flow neighborhoods and use the reconstruction error of an estimated flow vector to evaluate its reliability. In [24, a probabilistic model of flow patches is learned and approximated with a Gaussian distribution, which yields a confidence measure based on hypothesis testing. But this second class of confi- dence measures does not consider all aspects of flow uncertainty either, as the input images are not taken into account.

Recently, Mac Aodha et al. 30] proposed a confidence measure based on the input as well as the output of an optical flow algorithm. Using a multi-cue feature vector, a classifier is trained to predict whether the endpoint error at a certain pixel is smaller than a previously defined threshold. The probability output of the classifier is used as a confidence measure. However, 30] does not take advantage of uncertainty information available in the flow estimation procedure itself and requires a separate training step that is unnecessary for our model-inherent approach.

Uncertainty measures tailored to energy minimization approaches have been proposed especially for local energy methods, e.g., 3, 31, 40, 41. Model-inherent confidence measures designed for global energy formulations are quite rare, on the other hand. Kybic and Nieuwenhuis 27] introduce a method that relies on bootstrap resampling. Based on varying pixel contributions, repeated optical flow estimation is performed and a confidence measure is determined as the total standard deviation of the obtained flow predictions. Bruhn and Weickert [8] propose the inverse local energy as a confidence measure, which is applicable to a broad variety of energies. A large uncertainty is thus associated with a strong violation of the local model assumptions encoded in the energy formulation. Gehrig and Scharwächter 17 use the energy and combine it with several features such as the spatial and temporal flow variance in order to obtain a real-time confidence estimate. In contrast to the two above approaches, our method does not explicitly limit the receptive field of the confidence measure by only considering the local neighborhood of a pixel. Instead, uncertainty propagation is facilitated by means of iterative spatial inference.

In the past, several methods based on a probabilistic flow formulation have been proposed [35, 39, 40,41. However, many of these works are based on simplifying assumptions such as locally constant flow or a Gaussian distribution of brightness constancy errors. In [19, 26, 44, probabilistic approaches are applied to obtain improved models of optical flow. In contrast to our work, these methods do not apply a fully probabilistic approach, but fall back to a maximum aposteriori (MAP) estimate, i.e. minimize the underlying energy. Glocker et al. [18] use flow uncertainties in a dynamic Markov random field but rely on a discrete approach. The most closely related work of Chantas et al. 12] applies a variational-Bayes approach similar to ours. However, their method is not designed as a stand-alone flow algorithm, but only as an improved initialization in comparison to a simple Horn-Schunck approach. Therefore, the obtained results are not competitive with respect to the state of the art. Moreover, the paper does not take advantage of the probabilistic approach in the context of uncertainty measures. 


\section{Energy Framework}

Since our joint, model-inherent uncertainty estimation developed below is broadly applicable to different kinds of energy-based optical flow approaches 3, 15, 43, we first introduce a formalization that allows us to describe previously proposed optical flow methods in a unified manner.

In the following, we estimate optical flow between an image pair $I=\left\{I_{1}, I_{2}\right\}$ and denote the estimate as $\mathbf{y}=\left(\mathbf{y}_{i j}\right)_{i j}=\left(u_{i j}, v_{i j}\right)_{i j}^{\mathrm{T}}$ for pixels $(i, j), i=1, \ldots, n$, $j=1, \ldots, m$. Energy-based approaches estimate the optical flow as the minimizer $\mathbf{y}^{\star}$ of an energy function

$$
E(\mathbf{y} ; I)=E_{\mathrm{D}}(\mathbf{y} ; I)+\lambda_{\mathrm{S}} E_{\mathrm{S}}(\mathbf{y})
$$

with $E_{\mathrm{D}}(\mathbf{y} ; I)$ denoting a data term that encourages the flow to be consistent with input images $I_{1}$ and $I_{2}$. The spatial term $E_{\mathrm{S}}(\mathbf{y})$ imposes a (smoothness) prior on the flow, and $\lambda_{\mathrm{S}}$ represents a trade-off parameter between the terms.

In the following, we use functions $f_{\mathrm{D}}(\cdot)$ and $f_{\mathrm{S}}(\cdot)$ to formalize violations of the assumptions underlying the chosen optical flow model. For instance, the intensity difference of corresponding pixels in $I_{1}$ and $I_{2}$ may be evaluated to model a brightness constancy assumption for the data term. So-called penalty functions $\rho_{\mathrm{D}}(\cdot)$ and $\rho_{\mathrm{S}}(\cdot)$ penalize violations of the assumptions modeled in $f_{\mathrm{D}}(\cdot)$ and $f_{\mathrm{S}}(\cdot)$. The energy terms $E_{\mathrm{D}}$ and $E_{\mathrm{S}}$ can then be described as a sum of the contributions from all pixels such that

$$
\begin{aligned}
E_{\mathrm{D}}(\mathbf{y} ; I) & =\sum_{i, j} \rho_{\mathrm{D}}\left(f_{\mathrm{D}}\left(\mathbf{y}_{i j} ; I\right)\right) \\
E_{\mathrm{S}}(\mathbf{y}) & =\sum_{i, j} \sum_{\left(i^{\prime}, j^{\prime}\right) \in S(i, j)} \rho_{\mathrm{S}}\left(f_{\mathrm{S}}\left(\mathbf{y}_{i j}, \mathbf{y}_{i^{\prime} j^{\prime}}\right)\right)
\end{aligned}
$$

with $S(i, j)$ describing a set of neighbors of pixel $(i, j)$.

It has been shown that the estimated flow field $\mathbf{y}^{\star}$ can be improved by adding a non-local term to the energy function in Eq. 11 25, 43. Thus, we optionally use an additional non-local term $E_{\mathrm{N}}(\mathbf{y})$ akin to $E_{\mathrm{S}}(\mathbf{y})$, considering an extended neighborhood $N(i, j)$. Again, $E_{\mathrm{N}}(\mathbf{y})$ is described by a model assumption $f_{\mathrm{N}}(\cdot)$ and its corresponding penalty function $\rho_{\mathrm{N}}(\cdot)$.

Common choices for penalty functions $\rho(\cdot)$ are, e.g., a quadratic function 21, a Lorentzian function [5, or a (generalized) Charbonnier function 9,43. Many of these functions can be described by the negative logarithm of a Gaussian Scale Mixture (GSM) 44,,46. Thus, in the following we rely on this simple but powerful class of functions and represent the penalty terms as GSMs of $L$ components

$$
\rho(z)=-\log \left[\sum_{l=1}^{L} \pi_{l} \mathcal{N}\left(z ; 0, \sigma_{l}^{2}\right)\right]
$$

with $\mathcal{N}(z ; \mu, \sigma)$ being a normal distribution with mean $\mu=0$ and variance $\sigma=\sigma_{l}$; the weights $\pi_{l} \geq 0$ sum to 1 . As we will see, GSMs also benefit our probabilistic approach.

\section{Probabilistic Interpretation and Inference}

We now aim to approach optical flow estimation in a probabilistic manner. As the energy function $E(\mathbf{y} ; I)$ from Eq. (1) describes a Markov random field, it is easy to derive the corresponding posterior distribution in its Gibbs form as

$$
p(\mathbf{y} \mid I)=\frac{1}{Z} \exp \left\{-\frac{1}{T} E(\mathbf{y} ; I)\right\}
$$

with partition function $Z \equiv Z\left(I, T, \lambda_{\mathrm{S}}, \lambda_{\mathrm{N}}\right)$ and temperature $T$. In the following, w.l.o.g. we set $T=1$ and introduce an additional parameter $\lambda_{\mathrm{D}}$ scaling the data term $E_{\mathrm{D}}$.

To ease probabilistic inference, we use the same procedure as 43 and introduce an auxiliary flow field $\hat{\mathbf{y}}$ that allows us to decouple the non-local potential from the remaining terms of the posterior distribution in Eq. 5], i.e.

$$
\begin{aligned}
p(\mathbf{y}, \hat{\mathbf{y}} \mid I)=\frac{1}{Z} \exp \{ & -\lambda_{\mathrm{D}} E_{\mathrm{D}}(\mathbf{y} ; I)-\lambda_{\mathrm{S}} E_{\mathrm{S}}(\mathbf{y}) \\
& \left.-\lambda_{\mathrm{C}} E_{\mathrm{C}}(\mathbf{y}, \hat{\mathbf{y}})-\lambda_{\mathrm{N}} E_{\mathrm{N}}(\hat{\mathbf{y}})\right\}
\end{aligned}
$$

with $E_{\mathrm{C}}(\mathbf{y}, \hat{\mathbf{y}})=\sum_{i, j}\left\|\mathbf{y}_{i, j}-\hat{\mathbf{y}}_{i, j}\right\|_{2}^{2}$.

The log-posterior of Eq. 6) now has terms in the form of the logarithm of a sum of exponentials, which is challenging when deriving closed-form mean-field updates below. Here, we benefit from our choice to model each penalty function $\rho(\cdot)$ as a GSM. We follow 16, 28 to retain explicit latent variables $\mathbf{h}=\left(\mathbf{h}_{\mathrm{D}}, \mathbf{h}_{\mathrm{S}}, \mathbf{h}_{\mathrm{N}}\right)$, which are chosen to follow a discrete distribution and to have a 1-of-L representation. We then obtain an augmented penalty function $\rho_{\gamma}\left(z, \mathbf{h}_{\gamma}\right)$ with $\gamma \in\{\mathrm{D}, \mathrm{S}, \mathrm{N}\}$ as

$$
\rho_{\gamma}\left(z, \mathbf{h}_{\gamma}\right)=-\log \left[\prod_{l=1}^{L} \pi_{l}^{h_{\gamma, l}} \mathcal{N}\left(z ; 0, \sigma_{l}^{2}\right)^{h_{\gamma, l}}\right] .
$$

At this point, the posterior $p(\mathbf{y}, \hat{\mathbf{y}}, \mathbf{h} \mid I)$ represents the probabilistic equivalent of the energy $E(\mathbf{y} ; I)$ in Eq. 1.

In our probabilistic setup, the flow estimate $\mathbf{y}^{\star}$ is chosen to minimize the expected loss over $p(\mathbf{y}, \hat{\mathbf{y}}, \mathbf{h} \mid I)$, i.e.

$$
\mathbf{y}^{\star}=\underset{\tilde{\mathbf{y}}}{\arg \min } \mathbb{E}_{p(\mathbf{y}, \hat{\mathbf{y}}, \mathbf{h} \mid I)}[l(\mathbf{y}, \tilde{\mathbf{y}})]
$$

with $l(\cdot, \cdot)$ being a suitable loss function such as the Average End-Point Error (AEPE) 34. The desired uncertainty measure can be obtained by considering the marginal distribution $p\left(\mathbf{y}_{i j} \mid I\right)$ of the flow estimates. In particular, we propose to use the marginal entropy at every pixel as a modelinherent uncertainty estimate of the flow prediction.

Inference. To obtain a flow estimate and the underlying marginal distributions at every pixel, we rely on an approximate inference scheme. We use variational inference $45{ }^{2}$

\footnotetext{
${ }^{2}$ Not to be confused with variational formulations common in flow.
} 
and approximate $p(\mathbf{y}, \hat{\mathbf{y}}, \mathbf{h} \mid I)$ with the help of a tractable distribution $q(\mathbf{y}, \hat{\mathbf{y}}, \mathbf{h} ; \boldsymbol{\theta})$ and variational parameters $\boldsymbol{\theta}$.

Following a naive mean-field assumption, we choose the parametric distribution $q$ to be factorized over $\mathbf{y}, \hat{\mathbf{y}}$, as well as $\mathbf{h}$. The marginal distribution of flow vectors $\mathbf{y}_{i j}$ and $\hat{\mathbf{y}}_{i j}$ is assumed to be Gaussian, e.g.

$$
q\left(\mathbf{y}_{i j} ; \boldsymbol{\theta}\right) \sim \mathcal{N}\left(\mathbf{y}_{i j} ; \boldsymbol{\mu}_{i j}, \boldsymbol{\Sigma}_{i j}\right) .
$$

It is reasonable to assume the horizontal and vertical flow components to be uncorrelated 37]. Thus, the covariances $\boldsymbol{\Sigma}_{i j}$ are modeled as diagonal matrices. As in 16,28 , the distributions of the latent variables are chosen to be multinomial

$$
q\left(\mathbf{h}_{\gamma, i j} ; \boldsymbol{\theta}\right)=\prod_{l=1}^{L} k_{\gamma, i j, l}^{h_{\gamma, i j, l}}
$$

so that the parameters $k_{\gamma, i j, l} \geq 0$ satisfy $\sum_{l} k_{\gamma, i j, l}=1$. The approximating distribution $q$ is then given as

$$
\begin{gathered}
q(\mathbf{y}, \hat{\mathbf{y}}, \mathbf{h} ; \boldsymbol{\theta})=\prod_{i, j}\left[q\left(\mathbf{y}_{i j} ; \boldsymbol{\theta}\right) \cdot q\left(\hat{\mathbf{y}}_{i j} ; \boldsymbol{\theta}\right)\right. \\
\left.\cdot \prod_{\gamma \in\{\mathrm{D}, \mathrm{S}, \mathrm{N}\}} q\left(\mathbf{h}_{\gamma, i j} ; \boldsymbol{\theta}\right)\right]
\end{gathered}
$$

with $\boldsymbol{\theta}=\left\{\boldsymbol{\mu}_{i j}, \hat{\boldsymbol{\mu}}_{i j}, \boldsymbol{\Sigma}_{i j}, \hat{\boldsymbol{\Sigma}}_{i j}, \mathbf{k}_{\gamma, i j}\right\}_{i j, \gamma}$.

Suitable variational parameters $\boldsymbol{\theta}^{\star}$ of $q$ are determined such that the Kullback-Leibler (KL) divergence between $p$ and its approximating distribution $q$ is minimized, i.e.

$$
\begin{gathered}
\boldsymbol{\theta}^{\star}=\underset{\boldsymbol{\theta}}{\arg \min } D_{K L}(q(\mathbf{y}, \hat{\mathbf{y}}, \mathbf{h} ; \boldsymbol{\theta}) \mid p(\mathbf{y}, \hat{\mathbf{y}}, \mathbf{h} \mid I)) \\
=\underset{\boldsymbol{\theta}}{\arg \min } \\
\mathbb{E}_{q(\mathbf{y}, \hat{\mathbf{y}}, \mathbf{h} ; \boldsymbol{\theta})}[\log q(\mathbf{y}, \hat{\mathbf{y}}, \mathbf{h} ; \boldsymbol{\theta})] \\
-\mathbb{E}_{q(\mathbf{y}, \hat{\mathbf{y}}, \mathbf{h} ; \boldsymbol{\theta})}[\log p(\mathbf{y}, \hat{\mathbf{y}}, \mathbf{h} \mid I)] .
\end{gathered}
$$

Due to the usage of explicit latent variables $\mathbf{h}$, it is possible to compute the expectations in Eq. 13 in an analytic way. While the derivation of the corresponding equations is tedious, the individual steps are elementary; see supplemental material for a more detailed explanation of the procedure.

We now estimate the flow by replacing the posterior $p$ in Eq. 8) with its approximating distribution $q$. When performing Bayesian risk minimization of the AEPE, the optical flow prediction is obtained as $\mathbf{y}_{i j}^{\star}=\boldsymbol{\mu}_{i j}$ and, therefore, corresponds to the mode of the variational distribution $q$.

As $q(\mathbf{y}, \hat{\mathbf{y}}, \mathbf{h} ; \boldsymbol{\theta})$ was defined in a factorized way, the corresponding marginal distribution at pixel $(i, j)$ is given as $q\left(\mathbf{y}_{i j} ; \boldsymbol{\theta}\right)$ and our proposed model-inherent uncertainty measure can be obtained as the marginal entropy

$$
\Psi_{\text {ProbFlow }}=H\left(\mathbf{y}_{i j}\right)=\log \left(\operatorname{det}\left(\boldsymbol{\Sigma}_{i j}\right)\right)+\text { const. }
$$

\section{Specific Models}

We now apply our ProbFlow approach to two specific energy functions commonly used for optical flow estimation.

\subsection{Probabilistic Classic Flow}

We first consider a classical Horn-Schunck-based objective based on brightness constancy as given in 43. Following the common approach to use a first-order Taylor approximation for a linearization of the data term, we obtain

$$
\begin{aligned}
f_{\mathrm{D}}\left(\mathbf{y}_{i j} ; I\right)= & I_{2}\left(i+u_{i j}^{0}, j+v_{i j}^{0}\right)-I_{1}(i, j) \\
& +\nabla_{2} I_{2}\left(i+u_{i j}^{0}, j+v_{i j}^{0}\right)^{\mathrm{T}}\left(\mathbf{y}_{i j}-\mathbf{y}_{i j}^{0}\right),
\end{aligned}
$$

where $\mathbf{y}_{i j}^{0}$ is the point of approximation and $\nabla_{2} I_{2}$ denotes the spatial derivatives of $I_{2}$. The smoothness prior in 43 assumes small flow gradients over a 4-neighborhood of horizontal and vertical flow components. The non-local term encourages smoothness over a neighborhood of size $5 \times 5$. In both cases, the smoothness assumption is represented as

$$
f_{\mathrm{S}}\left(x_{i j}, x_{i^{\prime} j^{\prime}}\right)=f_{\mathrm{N}}\left(x_{i j}, x_{i^{\prime} j^{\prime}}\right)=x_{i j}-x_{i^{\prime} j^{\prime}} .
$$

Akin to energy minimization approaches 43 , we found the use of the non-local term crucial for obtaining accurate optical flow estimates, since mean-field inference using only the terms $E_{\mathrm{D}}$ and $E_{\mathrm{S}}$ is rather outlier-prone.

\subsection{Probabilistic FlowFields}

In a second setup, we aim for a probabilistic version of FlowFields 11. We follow Bailer et al. and consider the energy used in the post-processing step of EpicFlow 36, which uses a data term based on gradient constancy. As proposed in [4], the image gradient terms are normalized w.r.t. the spatial derivatives, which is helpful to avoid outliers in the flow field. We obtain the linearized data assumption as

$$
\begin{aligned}
f_{\mathrm{D}}\left(\mathbf{y}_{i j} ; I\right)= & \\
\| \sum_{r=1}^{3} \theta_{i j}^{r} \circ & {\left[\nabla_{2} I_{2}^{r}\left(i+u_{i j}^{0}, j+v_{i j}^{0}\right)-\nabla_{2} I_{1}^{r}(i, j)\right.} \\
& \left.+\mathbf{H}\left(I_{2}^{r}\left(i+u_{i j}^{0}, j+v_{i j}^{0}\right)\right)\left(\mathbf{y}_{i j}-\mathbf{y}_{i j}^{0}\right)\right] \|_{2}
\end{aligned}
$$

Here, $r=1, \ldots, 3$ indicates the RGB color channels, $\mathbf{H}\left(I_{2}^{r}\right)$ denotes the Hessian of $I_{2}^{r}$, and $\circ$ is the Hadamard product. The normalization coefficient $\theta_{i j}^{r}$ is given as

$$
\theta_{i j}^{r}=\left(\begin{array}{c}
\theta_{i j, 1}^{r} \\
\theta_{i j, 2}^{r}
\end{array}\right), \theta_{i j, k}^{r}=\frac{1}{\sqrt{\left\|\mathbf{H}\left(I_{2}^{r}\right) \cdot \mathbf{e}_{k}\right\|_{2}^{2}+\zeta^{2}}}
$$

with 2D unit vectors $\mathbf{e}_{k}$ and a small constant $\zeta>0$ [49].

The smoothness term of 36 is based on the flow gradient norm and uses additional filters of size $2 \times 3$ and $3 \times 2$ as described in 38 when calculating the flow derivatives. To keep the inference problem consistent, our approach differs slightly from 36 in that we only use the forward gradient filter described in Eq. 16, and obtain

$$
f_{\mathrm{S}}\left(\mathbf{y}_{i j}, \mathbf{y}_{i^{\prime} j^{\prime}}\right)=\sqrt{\left(u_{i j}-u_{i^{\prime} j^{\prime}}\right)^{2}+\left(v_{i j}-v_{i^{\prime} j^{\prime}}\right)^{2}}
$$


for $\mathbf{y}_{i^{\prime} j^{\prime}}$ in a 4-neighborhood of $\mathbf{y}_{i j}$.

Following [36, a locally adaptive trade-off parameter $\lambda_{\mathrm{S}}(\mathbf{x})=\exp \left(-\kappa\left\|\nabla_{2} I_{1}(\mathbf{x})\right\|\right)$ is used. Similar to MAP estimation, the minimization of the KL divergence in Eq. 13. obtained from $f_{\mathrm{D}}, f_{\mathrm{S}}$, and $\lambda_{\mathrm{S}}(\mathbf{x})$ performs well in practice. Therefore, an additional non-local term can be neglected.

\section{Implementation}

Optical flow estimation by energy minimization is known to be far from easy. Similarly, an application of mean-field inference is non-trivial in this context. Moreover, it is essential to consider several details commonly used with energy-based approaches to obtain satisfying results 15, 43. A summary of basic design choices as well as an extensive analysis of the influence of all described specifics can be found in the supplemental material.

Common per-pixel updates, e.g. [48], do not work well for the mean-field inference of Eq. 13). Instead, we keep the corresponding optimization procedure as efficient as possible and use a block-coordinate descent scheme updating flow estimates $\boldsymbol{\mu}$, variances $\boldsymbol{\Sigma}$, and latent variables $\mathbf{k}$ in an alternating manner. We derive the gradient of the KL divergence in Eq. (13), set it to zero, and obtain an update equation for each set of variables (see supplemental). As with MAP, we found a joint update of the flow predictions at all pixels to be crucial to obtaining smooth flow fields.

Parameters. To determine suitable trade-off parameters $\lambda$, it is not sufficient to follow the common approach and choose parameters that lead to the smallest AEPE on a training set 15, 43. Instead, we evaluate the quality of both the obtained flow predictions and the uncertainty measure in order to ensure accurate flow estimates and meaningful entropies. To assess the quality of our uncertainty measure, we follow the approaches in $8,24,27,30$ and compute so-called sparsification plots. To that end, the pixels of a flow field are sorted according to the estimated uncertainties. Subsequently, an increasing percentage of the pixels is removed and the AEPE of the remaining pixels is calculated. In order to evaluate how well different uncertainty measures perform on an entire dataset, we propose to normalize the graphs, calculate the area under curve (AUC), and average over the sequences. To consider the trade-off between flow accuracy and quality of the uncertainty measure, we evaluate an $\mathrm{F}_{1}$-score

$$
\mathrm{F}_{1}=\frac{\mathrm{AEPE} \cdot c \mathrm{AUC}}{\mathrm{AEPE}+c \mathrm{AUC}}
$$

over the training data with constant $c$ weighting the influence of the two metrics. We then determine parameters using Bayesian optimization 42$]$ of Eq. 20,

Concerning the penalty functions, we follow the approach in 44 and learn appropriate GSM models for data likelihood, smoothness prior, as well as the non-local term from the respective training datasets or a randomly chosen subset thereof. Using manually determined variances $\sigma_{l}$, a simple expectation maximization algorithm is used to obtain the corresponding weights $\pi_{l}$. We observe that GSMs with $L=10$ components perform well in practice. To save computational time, we resort to $L=5$ components for our probabilistic implementation of Classic Flow.

Details. For Probabilistic Classic Flow, we follow the underlying energy approach (ClassicA, [43]) and use zero flow as an initialization; we denote the method as ProbClassicA. Variances are initialized as $\sigma_{\text {init }}=1 \mathrm{e}-7$, latent variables as $k_{\text {init }}=1 / L$. During the inference process, the parameter $\lambda_{\mathrm{C}}$ is annealed as in 43. The parameters $\lambda_{\mathrm{D}}, \lambda_{\mathrm{S}}$, and $\lambda_{\mathrm{N}}$ are obtained with Bayesian optimization of the $\mathrm{F}_{1}$-score (Eq. 20 using $c=5$. As suggested by Sun $e t$ al. in the context of MAP, we use the variables $\hat{\mu}$ as the flow prediction. The corresponding uncertainties are then obtained from $\hat{\boldsymbol{\Sigma}}$.

For Probabilistic FlowFields, we use the state-of-the-art FlowFields method 11 to generate sparse matches. Following Bailer et al., we apply the EpicFlow 36 postprocessing step with Sintel parameters to interpolate the matches and use the results to initialize our algorithm. This method will be denoted as ProbFlowFields. Variances and latent variables are initialized as for ProbClassicA. Trade-off parameters $\lambda_{\mathrm{D}}, \lambda_{\mathrm{S}}$, and $\kappa$ are obtained again with Bayesian optimization [42]. The parameter of the $\mathrm{F}_{1}$ score (Eq. 20) is chosen as $c=100$ since the AEPE is significantly higher on Sintel. We will make code available for ProbClassicA as well as for ProbFlowFields.

\section{Experiments \& Results}

In the following, we evaluate our probabilistic flow approach and assess the quality of our uncertainty measure by comparing it to different approaches from the literature.

\subsection{Competing uncertainty measures}

We apply existing uncertainty measures on top of the corresponding energy minimization approaches (i.e. ClassicA or FlowFields) in order to analyze the benefit of our combined flow prediction and uncertainty estimation. We limit ourselves to a few select methods here and give a more extensive comparison in the supplemental material.

Barron et al. 3] suggest a confidence measure based on the spatial gradient of the input image. The corresponding uncertainty is obtained as $\Psi_{\text {Gradient }}=-\left\|\nabla_{2} I_{1}\right\|$ using central differences to approximate the gradient.

The learned confidence measure in 30 is based on a classifier that predicts with probability $\tilde{p}$ whether the error of the flow estimate at a certain pixel is smaller than a specified threshold $\epsilon_{T}$. We use the implementation of MacAodha et al. and train $\Psi_{\text {Learned }}=-\tilde{p}$ as described in 30. 


\begin{tabular}{lccccc}
\hline \multirow{2}{*}{ Method } & \multicolumn{2}{c}{ training } & & \multicolumn{2}{c}{ test } \\
\cline { 2 - 3 } \cline { 6 - 7 } \cline { 6 - 7 } & AEPE & rel. chg. & & AEPE & rel. chg. \\
\hline Classic++ [43] & $\mathbf{0 . 2 8 5}$ & $\mathbf{- 0 . 0 4}$ & & $\mathbf{0 . 4 0 6}$ & $\mathbf{- 0 . 0 7}$ \\
ClassicA [43] & 0.295 & $>-0.01$ & & - & - \\
ProbClassicA (ours) & 0.296 & 0.00 & & 0.435 & 0.00 \\
\hline
\end{tabular}

Table 1. Average end-point error (AEPE) and its relative change (rel. chg.) in comparison to ProbClassicA on Middlebury.

As proposed by Bruhn and Weickert [8], we use the local energy contribution of the MAP estimate $\mathbf{y}_{\text {MAP }}$ as a modelinherent uncertainty $\Psi_{\text {Energy }}=E\left(\mathbf{y}_{\mathrm{MAP}} ; I\right)$. We apply the non-linear version of the underlying energy function as it shows an improved performance [10]. A data penalty for out-of-boundary pixels is determined on the training set.

For an additional baseline, we perform a Laplace approximation of the posterior $p(\mathbf{y}, \hat{\mathbf{y}} \mid I)$ around $\mathbf{y}_{\mathrm{MAP}}$. With $\mathbf{H}$ denoting the Hessian of the linearized energy $E\left(\mathbf{y}_{\mathrm{MAP}} ; I\right)$, the covariance is given as $\boldsymbol{\Sigma}_{L}=\mathbf{H}^{-1}$. Similar to our approach, we obtain $\Psi_{\text {Laplace }}=-\log (\operatorname{det}(\mathbf{H}))+$ const.

Finally, we consider an oracle uncertainty measure $\Psi_{\text {Oracle }}$, for which the uncertainty of a pixel is given by its end-point error. Thus, the estimate $\Psi_{\text {Oracle }}$ provides a bound for the best possible uncertainty estimation. Note that we consider oracle uncertainties from the predictions obtained by MAP estimation; the oracle uncertainties for our flow predictions show a very similar behavior.

\subsection{ProbClassicA}

We first evaluate the application of ProbFlow to the ClassicA model described in Sec. 5.1. We rely on the Middlebury dataset 2], which has frequently been used to evaluate the performance of optical flow confidence measures. In order to compare different uncertainty approaches, ground truth optical flow is needed. As the Middlebury training set includes only 8 image pairs, we need to resort to training and testing the uncertainty measures on the same data.

To compare the performance of different flow estimation algorithms, we rely on the commonly used AEPE. Table 1 gives results on Middlebury training and test. Our method (ProbClassicA) performs on par with ClassicA. On the one hand, this is to be expected as both approaches share the same energy formulation and energy-based methods use highly elaborate schemes. On the other hand, this is the first time that a fully probabilistic method achieves competitive results on a public benchmark. For completeness, we also include the related Classic++, which shows slightly better results as its median filtering step allows for a more effective outlier suppression than an additional nonlocal term 25. The median filter cannot easily be applied to our case, but the results for ClassicA and ProbClassicA could be further improved by adding weights to the nonlocal term 43 .

The evaluation of uncertainty measures is more chal-

\begin{tabular}{lcccc}
\hline Uncertainty measure & AUC & rel. chg. & CC & rel. chg. \\
\hline Gradient [3] & 0.971 & 1.08 & 0.023 & 0.94 \\
Laplace & 0.656 & 0.41 & 0.160 & 0.57 \\
Energy [8] & 0.498 & 0.07 & 0.303 & 0.19 \\
Learned [30] & 0.496 & 0.06 & 0.324 & 0.13 \\
ProbClassicA (ours) & $\mathbf{0 . 4 6 6}$ & $\mathbf{0 . 0 0}$ & $\mathbf{0 . 3 7 4}$ & $\mathbf{0 . 0 0}$ \\
Oracle & 0.255 & - & 1.000 & - \\
\hline
\end{tabular}

Table 2. Area under curve (AUC), Spearman's rank correlation coefficient (CC), and relative change (rel. chg.) in comparison to our uncertainty measure on the Middlebury dataset.

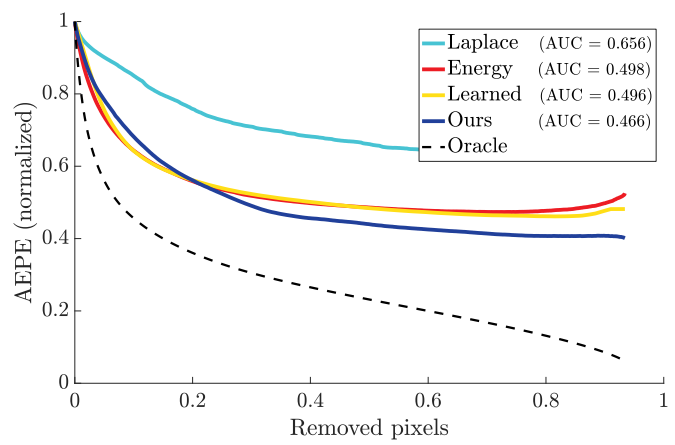

Figure 2. Sparsification plots averaged over Middlebury training.

lenging. Sparsification plots are commonly considered 8, 24, 27, 30. To be able to compare the performance of different uncertainty measures over an entire dataset, we calculate the AUC as described in Sec.6. However, as argued by Márquez-Valle et al. 31], sparsification plots do not allow to estimate how strongly an uncertainty estimate is related to the underlying pixel errors. To address this, we propose to compute the Spearman's rank correlation coefficient (CC), which estimates how well the examined uncertainty values can be mapped onto the corresponding endpoint errors using an arbitrary monotonic function.

Table 2 and the sparsification plots in Fig. 2 show that our uncertainty predictions are clearly superior in detecting the most reliable flow estimates. The gradient uncertainty has almost no ability to rank the pixels according to their accuracy. Hence, the simple consideration of input data appears insufficient. Similarly, the Laplace measure does not lead to satisfying results. $\Psi_{\text {Energy }}$ and $\Psi_{\text {Learned }}$ lead to very similar AUC results, whereby the learned uncertainty takes substantial time for training. Our method improves the AUC over previous ones by more than $6 \%$. Moreover, the evaluation of the $\mathrm{CC}$ reveals that our uncertainty measure shows by far the highest correlation between the assigned uncertainty value and the per-pixel end-point error with a relative improvement of $13 \%$ over the learned uncertainty.

\subsection{ProbFlowFields}

Next, we apply our probabilistic approach to the competitive FlowFields method as described in Sec.6. We use 


\begin{tabular}{lccccc}
\hline \multirow{2}{*}{ Method } & \multicolumn{2}{c}{ validation } & & \multicolumn{2}{c}{ test } \\
\cline { 2 - 3 } \cline { 6 - 7 } \cline { 6 - 7 } & AEPE & rel. chg. & & AEPE & rel. chg. \\
Initialization & 3.303 & 0.06 & & - & - \\
FlowFields [1] & 3.147 & $<0.01$ & & $5.727^{\dagger}$ & $<0.01$ \\
FlowFields & 3.161 & 0.01 & & - & - \\
ProbFlowFields (ours) & 3.127 & 0.00 & & 5.696 & 0.00 \\
ProbFlowFields + BS & $\mathbf{3 . 0 5 2}$ & $\mathbf{- 0 . 0 2}$ & & $\mathbf{5 . 6 2 8}$ & $\mathbf{- 0 . 0 1}$ \\
\hline
\end{tabular}

Table 3. Average end-point error (AEPE) and relative change (rel. chg.) w.r.t. to ProbFlowFields on Sintel. ${ }^{\dagger}$ FlowFields shows better results than the ones published on the website. See text for details.

\begin{tabular}{lcccc}
\hline Uncertainty measure & AUC & rel. chg. & CC & rel. chg. \\
\hline Gradient [3] & 1.022 & 1.57 & -0.009 & 1.02 \\
Laplace & 0.657 & 0.65 & 0.257 & 0.54 \\
Energy [8] & 0.470 & 0.18 & 0.434 & 0.23 \\
Learned [30] & 0.474 & 0.19 & 0.451 & 0.20 \\
ProbFlowFields (ours) & $\mathbf{0 . 3 9 8}$ & $\mathbf{0 . 0 0}$ & $\mathbf{0 . 5 6 3}$ & $\mathbf{0 . 0 0}$ \\
Oracle & 0.182 & - & 1.000 & - \\
\hline
\end{tabular}

Table 4. Area under curve (AUC), Spearman's rank correlation coefficient (CC), and relative change (rel. chg.) in comparison to our uncertainty measure on a Sintel benchmark validation set.

the more recent Sintel benchmark [11, which in comparison to the Middlebury dataset, allows to partition its more than 1000 flow sequences into training and validation sets. Exemplary flow and uncertainty estimates as well as the corresponding ground truth can be seen in Fig. 3 .

Table 3 summarizes the AEPEs on our validation set and the test set of the Sintel benchmark. For comparison, we also report results of FlowFields ${ }^{\star}$, based on the same consistent EpicFlow energy variant underlying ProbFlowFields, c.f. Sec. 5.2. Again, our estimates from ProbFlowFields are competitive with its underlying energy method and we observe results on par with the original FlowFields.

Evaluated on the Sintel test set, ProbFlowFields currently ranks $6^{\text {th }}$ in comparison to previously published methods. Please note that the FlowFields test results shown in Table 3 are superior to the publicly available AEPE of 5.810. To suppress the effect of the random component in the matches of FlowFields and thus have a fair comparison, we have re-evaluated the original FlowFields implementation using the exact same matches as for our approach.

The performance of the competing uncertainty measures is evaluated in Table 4. Again, the Gradient and Laplace uncertainties perform considerably worse than the remaining approaches. The measures $\Psi_{\text {Learned }}$ and $\Psi_{\text {Energy }}$ result in similar AUC values. Our method again leads to a clear improvement of over $18 \%$. Moreover, we also improve the CC by $20 \%$ in comparison to the second best measure. The sparsification plots in Fig. 4 show that our uncertainty measure leads to the best result for all fractions of removed pixels. Strikingly, the energy-based uncertainty as well as the
Laplace measure show a strong increase of the AEPE when only a small fraction of pixels are kept. This is clearly undesirable as it indicates that the optical flow predictions are incorrect for pixels that are considered as highly reliable. Our probabilistic approach does not show this behavior.

To illustrate the benefits of our uncertainties in postprocessing, we apply the fast bilateral solver 4 on top of ProbFlowFields, improving the AEPE by $2.4 \%$ and $1.2 \%$ on the validation and test set, respectively (c.f. Table 3 . In comparison, post-processing assuming equally reliable flow yields an improvement of only $0.4 \%$ on the validation set.

In a last experiment, we evaluated the overall runtime on our Sintel validation set (Intel Core i7-3930K, 3.2 GHz, 6 cores). The average runtimes are 19.9s for FlowFields and 38.1s for ProbFlowFields, i.e. the additional estimation of uncertainties has an overhead of $\sim 1.9 x$. The best post-hoc uncertainty measure (learned [30]) requires $123.8 \mathrm{~s}$ on average, thus takes significantly longer than our joint approach.

\subsection{Application to motion segmentation}

We now show the benefit of our uncertainty estimates when optical flow is used as a cue for motion segmentation. Current state-of-the-art methods (e.g. 22, 33]) build upon precomputed point trajectories, i.e. spatio-temporal curves that describe the individual point motion over extended periods. Ideally, such point trajectories are sampled with a regular density, they are long and reliable [6.

We build on the minimum cost multicut approach of 22 to obtain sparse motion segmentations. We follow Keuper et al. and generate point trajectories as in 6. In a first setup, we use FlowFields 11 to compute forward-backward (FB) flow for all consecutive image pairs. Subsequently, points are sampled on a regular grid in the first frame and tracked as long as (1) their FB flows are consistent, and (2) the gradient magnitude of the flow is below a threshold. If tracks are stopped, new points are inserted to preserve sampling regularity unless no points can be tracked in a region.

In a second setting, we apply ProbFlowFields using parameters trained on Sintel to compute FB flow along with normalized forward uncertainties $\Psi^{\mathrm{F}}$. Here, we keep the FB condition (1) and use a new condition (3) such that we end any track passing through location $(i, j)$ if the pixel uncertainty $\Psi_{i j}^{\mathrm{F}}$ falls below an empirically determined threshold.

Our results in terms of segmentation precision, recall, Fmeasure, as well as the achieved trajectory density on the FBMS-59 dataset 33 are given in Table 5. For both approaches, sparse trajectories are sampled at 8 pixel distance, and framewise dense segmentations are computed using the variational approach of [32]. Moreover, we compare to the current state-of-the-art 22] on FBMS-59, which is based on Large Displacement Optical Flow (LDOF) 7.

The evaluation of sparse segmentations shows that ProbFlow allows for a higher average point density of more 

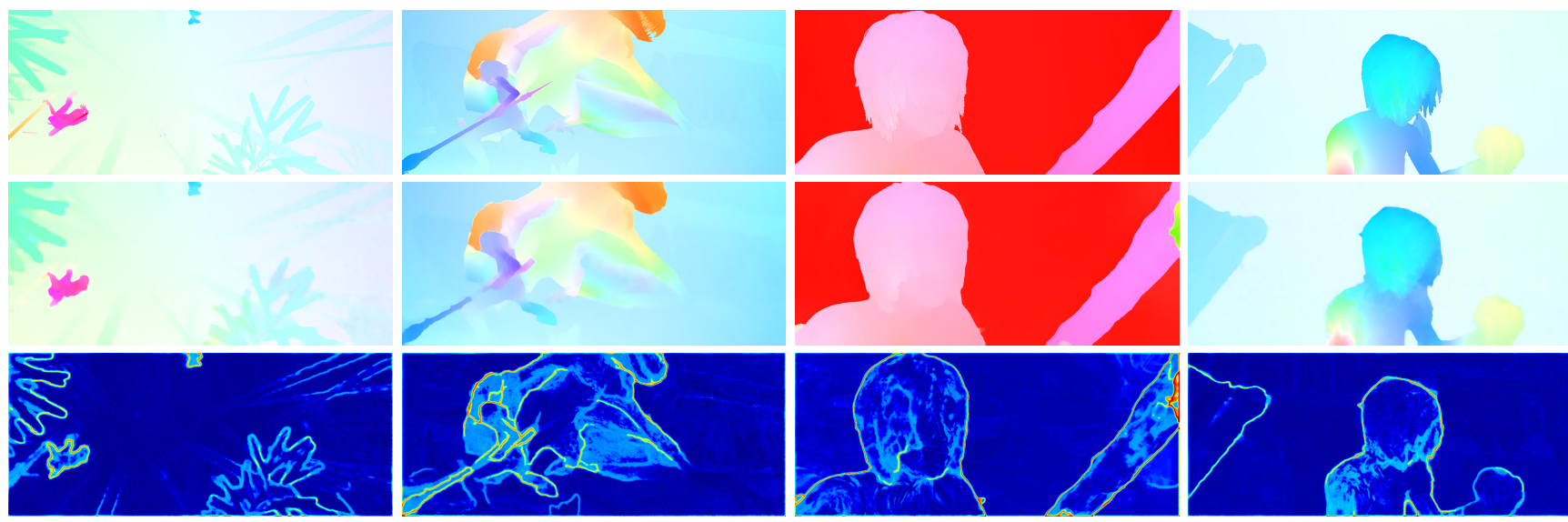

Figure 3. Examples of ground truth (top), flow predictions (middle), and uncertainty estimates (bottom) from ProbFlowFields on Sintel.

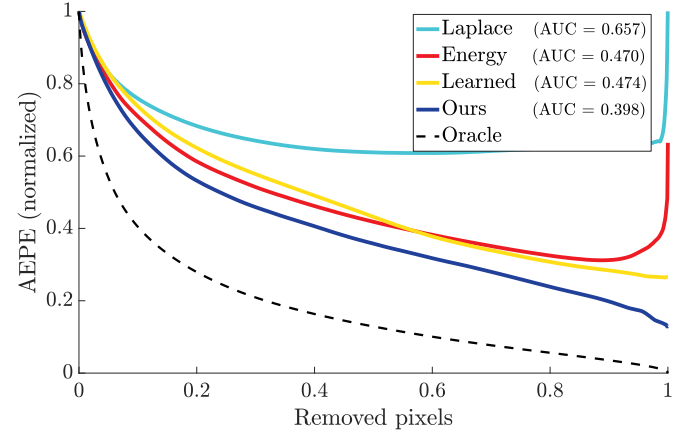

Figure 4. Sparsification plots averaged over Sintel validation.

than $1.18 \%$ compared to a maximal density of $0.89 \%$ with FlowFields 11 or LDOF 7 ]. Low point density usually indicates flow inconsistencies in homogeneous regions and therefore a more uneven sampling. As can be seen by the increased F-measure on sparse and especially on densified segmentations, the improvement in the trajectory computation directly translates to an improved segmentation quality.

To illustrate the complementarity of our uncertainties to the FB condition, we perform an experiment using ProbFlowFields estimates and the FB check (1), but not the corresponding uncertainties, i.e. omitting (3). In this case, the F-measure of the sparse matches drops significantly. Using FlowFields estimates and the post-hoc measure $\Psi_{\text {Energy }}$, the uncertainties again complement (1). However, the usage of ProbFlowFields yields a higher F-measure, thus showing a clear benefit also in this application.

\section{Conclusion}

To address the issue that optical flow estimates are not equally reliable throughout an image, we introduced ProbFlow - a probabilistic framework for the joint estimation of optical flow and its underlying uncertainty. Starting from conventional energy minimization methods, we derived the posterior distribution and used variational infer-

\begin{tabular}{lcccc}
\hline Training set (29 seq.) & $\mathrm{D}$ & $\mathrm{P}$ & $\mathrm{R}$ & $\mathrm{F}$ \\
\hline LDOF [7] & $0.81 \%$ & $86.73 \%$ & $73.08 \%$ & $79.32 \%$ \\
FlowFields (1+2) & $0.83 \%$ & $87.19 \%$ & $74.33 \%$ & $80.25 \%$ \\
FlowFields (1) & $1.17 \%$ & $85.10 \%$ & $71.36 \%$ & $77.63 \%$ \\
FlowFields + $\Psi_{\text {Energy }}$ & $1.17 \%$ & $84.62 \%$ & $72.57 \%$ & $78.13 \%$ \\
ProbFlowFields (1+3) & $1.18 \%$ & $\mathbf{8 7 . 6 8 \%}$ & $\mathbf{7 5 . 1 3 \%}$ & $\mathbf{8 0 . 9 2 \%}$ \\
ProbFlowFields (1) & $\mathbf{1 . 3 4 \%}$ & $84.96 \%$ & $72.14 \%$ & $78.03 \%$ \\
\hline FlowFields [1] dense & $100 \%$ & $86.14 \%$ & $67.28 \%$ & $75.55 \%$ \\
ProbFlowFields dense & $100 \%$ & $\mathbf{8 7 . 0 0 \%}$ & $\mathbf{7 0 . 1 5 \%}$ & $\mathbf{7 7 . 6 7 \%}$ \\
\hline Test set (30 seq.) & $\mathrm{D}$ & $\mathrm{P}$ & $\mathrm{R}$ & $\mathrm{F}$ \\
\hline LDOF [7] & $0.87 \%$ & $\mathbf{8 7 . 8 8 \%}$ & $67.70 \%$ & $76.48 \%$ \\
FlowFields (1+2) & $0.89 \%$ & $86.88 \%$ & $69.74 \%$ & $77.37 \%$ \\
ProbFlowFields (1+3) & $\mathbf{1 . 1 9 \%}$ & $84.99 \%$ & $\mathbf{7 2 . 8 3 \%}$ & $\mathbf{7 8 . 4 4 \%}$ \\
\hline FlowFields [1] dense & $100 \%$ & $84.38 \%$ & $61.03 \%$ & $70.83 \%$ \\
ProbFlowFields dense & $100 \%$ & $\mathbf{8 5 . 4 1 \%}$ & $\mathbf{6 6 . 9 3 \%}$ & $\mathbf{7 5 . 0 5 \%}$ \\
\hline
\end{tabular}

Table 5. Motion segmentation results on the FBMS-59 dataset. We report point density (D), average precision (P), average recall $(\mathrm{R})$, and F-measure. All results are computed for a sparse trajectory sampling at 8 pixel distance with MCe motion segmentation 22]. All results for LDOF are taken from 22].

ence to estimate the flow and a model-inherent uncertainty. This is unlike existing uncertainty measures that detect regions of unreliable flow in a post-hoc step. We applied our approach to two different energy formulations on the Middlebury and Sintel benchmarks, where we obtain competitive flow estimates and significantly improved uncertainties. Applying our uncertainty estimates in the context of motion segmentation, we were able to discard erroneous flow estimates and generate highly reliable point trajectories.

Acknowledgments. The research leading to these results has received funding from the European Research Council under the European Union's Seventh Framework Programme (FP/20072013)/ERC Grant agreement No. 307942. We would like to thank Tobias Plötz and Jochen Gast for helpful discussions. 


\section{References}

[1] C. Bailer, B. Taetz, and D. Stricker. Flow fields: Dense correspondence fields for highly accurate large displacement optical flow estimation. ICCV, 2015. 2, 4, 5, 7,8

[2] S. Baker, D. Scharstein, J. P. Lewis, S. Roth, M. J. Black, and R. Szeliski. A database and evaluation methodology for optical flow. Int. J. Comput. Vision, 92(1):1-31, 2011. 2,6

[3] J. L. Barron, D. J. Fleet, and S. S. Beauchemin. Performance of optical flow techniques. Int. J. Comput. Vision, 12(1):4377, 1994. 1, 2, 3, 5,6,7

[4] J. T. Barron and B. Poole. The fast bilateral solver. ECCV, 2016. 1,7

[5] M. J. Black and P. Anandan. The robust estimation of multiple motions: Parametric and piecewise-smooth flow fields. Comput. Vis. Image Und., 63(1):75-104, 1996. 1,3

[6] T. Brox and J. Malik. Object segmentation by long term analysis of point trajectories. ECCV, 2010.7

[7] T. Brox and J. Malik. Large displacement optical flow: Descriptor matching in variational motion estimation. IEEE T. Pattern Anal. Mach. Intell., 33(3):500 - 513, 2011. 7,8

[8] A. Bruhn and J. Weickert. A confidence measure for variational optic flow methods. In R. Klette, R. Kozera, L. Noakes, and J. Weickert, editors, Geometric Properties for Incomplete Data, pp. 283-298. Springer, 2006. 2, 5, 6, 7

[9] A. Bruhn, J. Weickert, and C. Schnörr. Lucas/Kanade meets Horn/Schunck: Combining local and global optic flow methods. Int. J. Comput. Vision, 61(3):211-231, 2005. 1,3

[10] M. Brumm, J. M. Marcinczak, and R. Grigat. Improved confidence measures for variational optical flow. VISAPP, 2015. 6

[11] D. J. Butler, J. Wulff, G. B. Stanley, and M. J. Black. A naturalistic open source movie for optical flow evaluation. ECCV, 2012.2,7

[12] G. K. Chantas, T. Gkamas, and C. Nikou. Variational-Bayes optical flow. J. Math. Imaging Vision, 50(3):199-213, 2014. 2

[13] Q. Chen and V. Koltun. Full flow: Optical flow estimation by global optimization over regular grids. $C V P R, 2016.2$

[14] A. Dosovitskiy, P. Fischer, E. Ilg, P. Häusser, C. Hazırbaş, V. Golkov, P. v.d. Smagt, D. Cremers, and T. Brox. Flownet: Learning optical flow with convolutional networks. ICCV, 2015. 1,2

[15] D. Fortun, P. Bouthemy, and C. Kervrann. Optical flow modeling and computation: A survey. Comput. Vis. Image Und., 134:1-21, 2015. 1,2,3,5

[16] J. Gast, A. Sellent, and S. Roth. Parametric object motion from blur. $C V P R, 2016.3,4$

[17] S. K. Gehrig and T. Scharwächter. A real-time multi-cue framework for determining optical flow confidence. ICCV Workshops, 2011.2

[18] B. Glocker, N. Paragios, N. Komodakis, G. Tziritas, and N. Navab. Optical flow estimation with uncertainties through dynamic MRFs. CVPR, 2008. 2

[19] V. M. Govindu. Revisiting the brightness constraint: Probabilistic formulation and algorithms. ECCV, 2006.2
[20] H. Haußecker and H. Spies. Motion. In B. Jähne, H. Haußecker, and P. Geißler, editors, Handbook of Computer Vision and Applications, vol. 2. Academic Press, 1999. 1,2

[21] B. K. P. Horn and B. G. Schunck. Determining optical flow. Artificial Intelligence, 17(1-3):185-203, 1981. 1,2,3

[22] M. Keuper, B. Andres, and T. Brox. Motion trajectory segmentation via minimum cost multicuts. ICCV, 2015. 2, 7, 8

[23] C. Kondermann, D. Kondermann, B. Jähne, and C. Garbe. An adaptive confidence measure for optical flows based on linear subspace projections. DAGM, 2007.2

[24] C. Kondermann, R. Mester, and C. Garbe. A statistical confidence measure for optical flows. $E C C V, 2008.1,2,5,6$

[25] P. Krähenbühl and V. Koltun. Efficient nonlocal regularization for optical flow. $E C C V, 2012.3,6$

[26] K. Krajsek and R. Mester. Bayesian model selection for optical flow estimation. DAGM, 2007.2

[27] J. Kybic and C. Nieuwenhuis. Bootstrap optical flow confidence and uncertainty measure. Comput. Vis. Image Und., 115(10):1449-1462, 2011. 1, 2, 5,6

[28] A. Levin, Y. Weiss, F. Durand, and W. T. Freeman. Efficient marginal likelihood optimization in blind deconvolution. CVPR, 2011. 3,4

[29] Y. Li, D. Min, M. S. Brown, M. N. Do, and J. Lu. SPMBP: Sped-up PatchMatch belief propagation for continuous MRFs. ICCV, 2015.2

[30] O. Mac Aodha, A. Humayun, M. Pollefeys, and G. J. Brostow. Learning a confidence measure for optical flow. IEEE T. Pattern Anal. Mach. Intell., 35(5):1107-1120, 2013. 1,2, 5, 6,7

[31] P. Márquez-Valle, D. Gil, and A. Hernàndez-Sabaté. A complete confidence framework for optical flow. ECCV Workshops and Demonstrations, 2012. 2, 6

[32] P. Ochs and T. Brox. Object segmentation in video: A hierarchical variational approach for turning point trajectories into dense regions. ICCV, 2011. 7

[33] P. Ochs, J. Malik, and T. Brox. Segmentation of moving objects by long term video analysis. IEEE T. Pattern Anal. Mach. Intell., 36(6):1187 - 1200, 2014. 1,7

[34] M. Otte and H.-H. Nagel. Optical flow estimation: Advances and comparisons. ECCV, 1994. 3

[35] D. Piao, P. G. Menon, and O. J. Mengshoel. Computing probabilistic optical flow using Markov random fields. Computational Modeling of Objects Presented in Images. Fundamentals, Methods, and Applications, pp. 241-247. Springer, 2014.2

[36] J. Revaud, P. Weinzaepfel, Z. Harchaoui, and C. Schmid. EpicFlow: Edge-preserving interpolation of correspondences for optical flow. $C V P R, 2015.1,2,4,5$

[37] S. Roth and M. J. Black. On the spatial statistics of optical flow. Int. J. Comput. Vision, 74(1):33-50, 2007. 4

[38] S. Roth and M. J. Black. Steerable random fields. ICCV, 2007. 4

[39] S. Roy and V. Govindu. MRF solutions for probabilistic optical flow formulations. ICPR, 2000. 2 
[40] E. P. Simoncelli, E. H. Adelson, and D. J. Heeger. Probability distributions of optical flow. CVPR, 1991.2

[41] A. Singh. An estimation-theoretic framework for image-flow computation. ICCV, 1990. 2

[42] J. Snoek, H. Larochelle, and R. P. Adams. Practical Bayesian optimization of machine learning algorithms. NIPS*2012, pp. 2951-2959.5

[43] D. Sun, S. Roth, and M. J. Black. A quantitative analysis of current practices in optical flow estimation and the principles behind them. Int. J. Comput. Vision, 106(2):115-137, 2014. $1,2,3,4,5,6$

[44] D. Sun, S. Roth, J. P. Lewis, and M. J. Black. Learning optical flow. $E C C V, 2008.2,3,5$
[45] M. J. Wainwright and M. I. Jordan. Graphical models, exponential families, and variational inference. Foundations and Trends in Machine Learning, 1(1-2):1-305, 2008. 3

[46] M. J. Wainwright and E. P. Simoncelli. Scale mixtures of gaussians and the statistics of natural images. NIPS $* 1999.3$

[47] A. Wedel, A. Meißner, C. Rabe, U. Franke, and D. Cremers. Detection and segmentation of independently moving objects from dense scene flow. EMMCVPR, 2009. 1

[48] J. Winn and C. M. Bishop. Variational message passing. J. Mach. Learn. Res., 6:661-694, 2005. 5

[49] H. Zimmer, A. Bruhn, and J. Weickert. Optic flow in harmony. Int. J. Comput. Vision, 93(3):368-388, 2011.4 


\title{
ProbFlow: Joint Optical Flow and Uncertainty Estimation - Supplemental Material -
}

\author{
Anne S. Wannenwetsch ${ }^{1}$ \\ Margret Keuper ${ }^{2}$ \\ Stefan Roth $^{1}$ \\ ${ }^{1}$ TU Darmstadt \\ ${ }^{2}$ University of Mannheim
}

Preface. In this supplemental material we derive update equations for the mean-field inference in Eq. 13. and show a proof for the solution of the Bayesian risk minimization in Eq. 8). We give further implementation details of ProbClassicA and ProbFlowFields, and present an analysis considering different design choices. Finally, we evaluate the performance of additional uncertainty measures and apply, for completeness, ProbFlowFields on the Middlebury benchmark [2].

\section{A. Mean-field Update Equations}

In the following, we show how to derive the mean-field update equations for ProbClassicA. Update equations for ProbFlowFields can be obtained similarly.

Notation. Note that strictly speaking the latent variables are given as $\mathbf{h}=\left(\mathbf{h}_{\gamma, i j, l}\right)_{\gamma, i j}$ with $\gamma \in\left\{\mathrm{D}, \mathrm{S}_{1}, \ldots, \mathrm{S}_{p}, \mathbf{N}_{1}, \ldots, \mathbf{N}_{q}\right\}$, $p=|S(i, j)|, q=|N(i, j)|$ as we have separate latent variables for all penalty functions. In the following, we therefore use the notation $\mathbf{h}_{S_{e}}, \mathbf{h}_{N_{e}}$ and $f_{S_{e}}(\cdot), f_{N_{e}}(\cdot)$ for $e \in S(i, j)$ and $e \in N(i, j)$, respectively. The flow vector of the corresponding neighboring pixel is denoted as $\mathbf{y}_{e}$. Moreover, GSM parameters $\pi_{l}$ and $\sigma_{l}$ differ for data, smoothness, and non-local potentials. For better readability, we drop indices D, S, and N that explicitly distinguish between the different GSMs.

Variational objective. As shown in Eq. 13, variational parameters $\boldsymbol{\theta}^{\star}$ are determined to minimize the Kullback-Leibler divergence between posterior $p$ and its approximating distribution $q$, i.e.

$$
\begin{aligned}
\boldsymbol{\theta}^{\star} & =\underset{\boldsymbol{\theta}}{\arg \min } D_{K L}(q(\mathbf{y}, \hat{\mathbf{y}}, \mathbf{h} ; \boldsymbol{\theta}) \mid p(\mathbf{y}, \hat{\mathbf{y}}, \mathbf{h} \mid I)) \\
& =\underset{\boldsymbol{\theta}}{\arg \min } \underbrace{\mathbb{E}_{q(\mathbf{y}, \hat{\mathbf{y}}, \mathbf{h} ; \boldsymbol{\theta})}[\log q(\mathbf{y}, \hat{\mathbf{y}}, \mathbf{h} ; \boldsymbol{\theta})]}_{\text {(1) entropy term }}-\underbrace{\mathbb{E}_{q(\mathbf{y}, \hat{\mathbf{y}}, \mathbf{h} ; \boldsymbol{\theta})}[\log p(\mathbf{y}, \hat{\mathbf{y}}, \mathbf{h} \mid I)]}_{\text {(2) }} .
\end{aligned}
$$

Recall that we defined the variational distribution $q$ in Eq. 11] as

$$
q(\mathbf{y}, \hat{\mathbf{y}}, \mathbf{h} ; \boldsymbol{\theta})=\prod_{i, j}\left[q\left(\mathbf{y}_{i j} ; \boldsymbol{\theta}\right) \cdot q\left(\hat{\mathbf{y}}_{i j} ; \boldsymbol{\theta}\right) \cdot \prod_{\gamma} q\left(\mathbf{h}_{\gamma, i j} ; \boldsymbol{\theta}\right)\right] .
$$

Then, the entropy term in (1) can be split up as follows:

$$
\begin{aligned}
\mathbb{E}_{q(\mathbf{y}, \hat{\mathbf{y}}, \mathbf{h} ; \boldsymbol{\theta})} & {[\log q(\mathbf{y}, \hat{\mathbf{y}}, \mathbf{h} ; \boldsymbol{\theta})]=\mathbb{E}_{q(\mathbf{y}, \hat{\mathbf{y}}, \mathbf{h} ; \boldsymbol{\theta})}\left[\sum_{i, j} \log q\left(\mathbf{y}_{i j} ; \boldsymbol{\theta}\right)+\sum_{i, j} \log q\left(\hat{\mathbf{y}}_{i j} ; \boldsymbol{\theta}\right)+\sum_{i, j} \sum_{\gamma} \log q\left(\mathbf{h}_{\gamma, i j} ; \boldsymbol{\theta}\right)\right] } \\
= & \sum_{i, j} \mathbb{E}_{q\left(\mathbf{y}_{i j} ; \boldsymbol{\theta}\right)} \log q\left(\mathbf{y}_{i j} ; \boldsymbol{\theta}\right)+\sum_{i, j} \mathbb{E}_{q\left(\hat{\mathbf{y}}_{i j} ; \boldsymbol{\theta}\right)} \log q\left(\hat{\mathbf{y}}_{i j} ; \boldsymbol{\theta}\right)+\sum_{i, j} \sum_{\gamma} \mathbb{E}_{q\left(\mathbf{h}_{\gamma, i j} ; \boldsymbol{\theta}\right)} \log q\left(\mathbf{h}_{\gamma, i j} ; \boldsymbol{\theta}\right) .
\end{aligned}
$$

Using the well-known entropy of a Gaussian and a multinomial distribution, we obtain

$$
\mathbb{E}_{q(\mathbf{y}, \hat{\mathbf{y}}, \mathbf{h} ; \boldsymbol{\theta})}[\log q(\mathbf{y}, \hat{\mathbf{y}}, \mathbf{h} ; \boldsymbol{\theta})]=-\frac{1}{2} \sum_{i, j} \log \left(\operatorname{det}\left(\boldsymbol{\Sigma}_{i j}\right)\right)-\frac{1}{2} \sum_{i, j} \log \left(\operatorname{det}\left(\hat{\boldsymbol{\Sigma}}_{i j}\right)\right)+\sum_{i, j} \sum_{\gamma} \sum_{l} k_{\gamma, i j, l} \log k_{\gamma, i j, l}+\text { const. }
$$


In order to evaluate the term (2) in Eq. 22b, it is necessary to compute

$$
\begin{aligned}
& \log p(\mathbf{y}, \hat{\mathbf{y}}, \mathbf{h} \mid I) \\
& =\log \left(\frac{1}{Z} \prod_{i, j}\left[\prod_{l} \pi_{l}^{h_{\mathrm{D}, i j, l}} \mathcal{N}\left(f_{\mathrm{D}}\left(\mathbf{y}_{i j} ; I\right) ; 0, \sigma_{l}^{2}\right)^{h_{\mathrm{D}, i j, l}}\right]^{\lambda_{\mathrm{D}}} \cdot \prod_{e \in S(i, j)}\left[\prod_{l} \pi_{l}^{h_{\mathrm{S}_{e}, i j, l}} \mathcal{N}\left(f_{\mathrm{S}_{e}}\left(\mathbf{y}_{i j}, \mathbf{y}_{e}\right) ; 0, \sigma_{l}^{2}\right)^{h_{\mathrm{S}_{e}, i j, l}}\right]^{\lambda_{\mathrm{S}}}\right. \\
& \left.\cdot \exp \left[-f_{\mathrm{C}}\left(\mathbf{y}_{i j}, \hat{\mathbf{y}}_{i j}\right)^{2}\right]^{\lambda_{\mathrm{C}}} \cdot \prod_{e \in N(i, j)}\left[\prod_{l} \pi_{l}^{h_{\mathrm{N}_{e}, i j, l}} \mathcal{N}\left(f_{\mathrm{N}_{e}}\left(\hat{\mathbf{y}}_{i j}, \hat{\mathbf{y}}_{e}\right) ; 0, \sigma_{l}^{2}\right)^{h_{\mathrm{N}_{e}, i j, l}}\right]^{\lambda_{\mathrm{N}}}\right) \\
& =\sum_{i, j}\left[\lambda_{\mathrm{D}} \sum_{l} h_{\mathrm{D}, i j, l}\left(\log \pi_{l}+\log \mathcal{N}\left(f_{\mathrm{D}}\left(\mathbf{y}_{i j} ; I\right) ; 0, \sigma_{l}^{2}\right)\right)+\lambda_{\mathrm{S}} \sum_{e \in S(i, j)} \sum_{l} h_{\mathrm{S}_{e}, i j, l}\left(\log \pi_{l}+\log \mathcal{N}\left(f_{\mathrm{S}_{e}}\left(\mathbf{y}_{i j}, \mathbf{y}_{e}\right) ; 0, \sigma_{l}^{2}\right)\right)\right. \\
& \left.-\lambda_{C} f_{\mathrm{C}}\left(\mathbf{y}_{i j}, \hat{\mathbf{y}}_{i j}\right)^{2}+\lambda_{\mathrm{N}} \sum_{e \in N(i, j)} \sum_{l} h_{\mathrm{N}_{e}, i j, l}\left(\log \pi_{l}+\log \mathcal{N}\left(f_{\mathrm{N}_{e}}\left(\hat{\mathbf{y}}_{i j}, \hat{\mathbf{y}}_{e}\right) ; 0, \sigma_{l}^{2}\right)\right)\right]-\log Z \\
& =\sum_{i, j}\left[\lambda_{\mathrm{D}} \sum_{l} h_{\mathrm{D}, i j, l}\left(\log \pi_{l}-\log \sigma_{l}-\frac{f_{\mathrm{D}}\left(\mathbf{y}_{i j} ; I\right)^{2}}{2 \sigma_{l}^{2}}\right)+\lambda_{\mathrm{S}} \sum_{e \in S(i, j)} \sum_{l} h_{\mathrm{S}_{e}, i j, l}\left(\log \pi_{l}-\log \sigma_{l}-\frac{f_{\mathrm{S}_{e}}\left(\mathbf{y}_{i j}, \mathbf{y}_{e}\right)^{2}}{2 \sigma_{l}^{2}}\right)\right. \\
& \left.-\lambda_{\mathrm{C}} f_{\mathrm{C}}\left(\mathbf{y}_{i j}, \hat{\mathbf{y}}_{i j}\right)^{2}+\lambda_{\mathrm{N}} \sum_{e \in N(i, j)} \sum_{l} h_{\mathrm{N}_{e}, i j, l}\left(\log \pi_{l}-\log \sigma_{l}-\frac{f_{\mathrm{N}_{e}}\left(\hat{\mathbf{y}}_{i j}, \hat{\mathbf{y}}_{e}\right)^{2}}{2 \sigma_{l}^{2}}\right)\right]+\mathrm{const}
\end{aligned}
$$

where we have defined $f_{\mathrm{C}}\left(\mathbf{y}_{i j}, \hat{\mathbf{y}}_{i j}\right)=\left\|\mathbf{y}_{i j}-\hat{\mathbf{y}}_{i j}\right\|_{2}$. We now take the expectation over $\mathbf{h}$ and simplify the remaining expectations as

$$
\begin{aligned}
& \mathbb{E}_{q(\mathbf{y}, \hat{\mathbf{y}}, \mathbf{h} ; \boldsymbol{\theta})} \log p(\mathbf{y}, \hat{\mathbf{y}}, \mathbf{h} \mid I) \\
& =\mathbb{E}_{q(\mathbf{y}, \hat{\mathbf{y}} ; \boldsymbol{\theta})} \sum_{i, j}\left[\lambda_{\mathrm{D}} \sum_{l} k_{\mathrm{D}, i j, l}\left(\log \pi_{l}-\log \sigma_{l}-\frac{f_{\mathrm{D}}\left(\mathbf{y}_{i j} ; I\right)^{2}}{2 \sigma_{l}^{2}}\right)\right. \\
& +\lambda_{\mathrm{S}} \sum_{e \in S(i, j)} \sum_{l} k_{\mathrm{S}_{e}, i j, l}\left(\log \pi_{l}-\log \sigma_{l}-\frac{f_{\mathrm{S}_{e}}\left(\mathbf{y}_{i j}, \mathbf{y}_{e}\right)^{2}}{2 \sigma_{l}^{2}}\right) \\
& \left.-\lambda_{\mathrm{C}} f_{\mathrm{C}}\left(\mathbf{y}_{i j}, \hat{\mathbf{y}}_{i j}\right)^{2}+\lambda_{\mathrm{N}} \sum_{e \in N(i, j)} \sum_{l} k_{\mathrm{N}_{e}, i j, l}\left(\log \pi_{l}-\log \sigma_{l}-\frac{f_{\mathrm{N}_{e}}\left(\hat{\mathbf{y}}_{i j}, \hat{\mathbf{y}}_{e}\right)^{2}}{2 \sigma_{l}^{2}}\right)\right]+\mathrm{const} \\
& =\sum_{i, j}\left[\lambda_{\mathrm{D}} \sum_{l} k_{\mathrm{D}, i j, l}\left(\log \pi_{l}-\log \sigma_{l}\right)+\lambda_{\mathrm{S}} \sum_{e \in S(i, j)} \sum_{l} k_{\mathrm{S}_{e}, i j, l}\left(\log \pi_{l}-\log \sigma_{l}\right)\right. \\
& \left.+\lambda_{\mathrm{N}} \sum_{e \in N(i, j)} \sum_{l} k_{\mathrm{N}_{e}, i j, l}\left(\log \pi_{l}-\log \sigma_{l}\right)\right]
\end{aligned}
$$

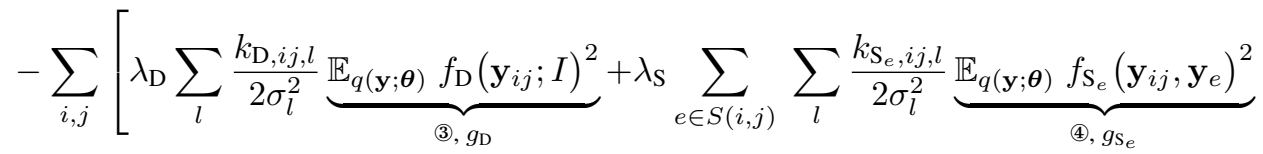

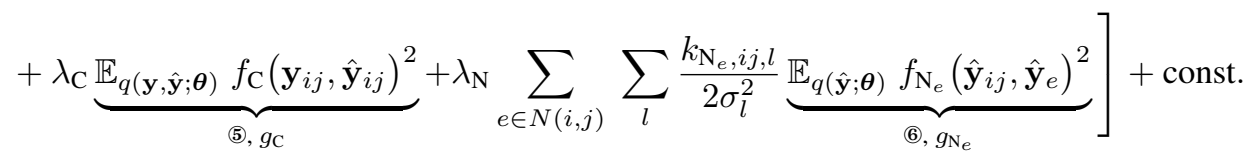


To solve the expectation value w.r.t. the linearized brightness constancy in (3), we define $a=I_{2}\left(i+u_{i j}^{0}, j+v_{i j}^{0}\right)-I_{1}(i, j)$ and $\mathbf{b}=\nabla_{2} I_{2}\left(i+u_{i j}^{0}, j+v_{i j}^{0}\right)^{\mathrm{T}}$. Then we have that

$$
\begin{aligned}
g_{\mathrm{D}}\left(\boldsymbol{\mu}_{i j}, \boldsymbol{\Sigma}_{i j} ; I\right) & =\mathbb{E}_{q(\mathbf{y} ; \boldsymbol{\theta})} f_{\mathrm{D}}\left(\mathbf{y}_{i j} ; I\right)^{2} \\
& =\mathbb{E}_{q\left(\mathbf{y}_{i j} ; \boldsymbol{\theta}\right)}\left[\left(a+\mathbf{b}^{\mathrm{T}}\left(\mathbf{y}_{i j}-\mathbf{y}_{i j}^{0}\right)\right)^{2}\right] \\
& =\mathbb{E}_{q\left(\mathbf{y}_{i j} ; \boldsymbol{\theta}\right)}\left[a^{2}+2 a \mathbf{b}^{\mathrm{T}}\left(\mathbf{y}_{i j}-\mathbf{y}_{i j}^{0}\right)+\left(\mathbf{y}_{i j}-\mathbf{y}_{i j}^{0}\right)^{\mathrm{T}}\left(\mathbf{b b}^{\mathrm{T}}\right)\left(\mathbf{y}_{i j}-\mathbf{y}_{i j}^{0}\right)\right] \\
& =a^{2}+2 a \mathbf{b}^{\mathrm{T}}\left(\boldsymbol{\mu}_{i j}-\mathbf{y}_{i j}^{0}\right)+\left(\boldsymbol{\mu}_{i j}-\mathbf{y}_{i j}^{0}\right)^{\mathrm{T}}\left(\mathbf{b b}^{\mathrm{T}}\right)\left(\boldsymbol{\mu}_{i j}-\mathbf{y}_{i j}^{0}\right)+\operatorname{Tr}\left(\mathbf{b} \mathbf{b}^{\mathrm{T}} \boldsymbol{\Sigma}_{i, j}\right) .
\end{aligned}
$$

We solve the expectation value $g_{\mathrm{S}_{e}}$ in (4) for an exemplary function $f_{\mathrm{S}_{e}}\left(\mathbf{y}_{i j}, \mathbf{y}_{e}\right)=u_{i j}-u_{e}$. All remaining terms as well as the terms $g_{\mathrm{N}_{e}}$ in (6) can be resolved in the same manner. Using $\mathbf{A}_{1}=\left(\begin{array}{ll}1 & 0 \\ 0 & 0\end{array}\right)$, it holds that

$$
\begin{aligned}
g_{\mathrm{S}_{e}}\left(\boldsymbol{\mu}_{i j}, \boldsymbol{\Sigma}_{i j}, \boldsymbol{\mu}_{e}, \boldsymbol{\Sigma}_{e}\right) & =\mathbb{E}_{q(\mathbf{y} ; \boldsymbol{\theta})} f_{\mathrm{S}_{e}}\left(\mathbf{y}_{i j}, \mathbf{y}_{e}\right)^{2} \\
& =\mathbb{E}_{q\left(\mathbf{y}_{e} ; \boldsymbol{\theta}\right)} \mathbb{E}_{q\left(\mathbf{y}_{i j} ; \boldsymbol{\theta}\right)}\left[\left(\mathbf{y}_{i j}-\mathbf{y}_{e}\right)^{\mathrm{T}} \mathbf{A}_{1}\left(\mathbf{y}_{i j}-\mathbf{y}_{e}\right)\right] \\
& =\mathbb{E}_{q\left(\mathbf{y}_{e} ; \boldsymbol{\theta}\right)}\left[\left(\boldsymbol{\mu}_{i j}-\mathbf{y}_{e}\right)^{\mathrm{T}} \mathbf{A}_{1}\left(\boldsymbol{\mu}_{i j}-\mathbf{y}_{e}\right)+\operatorname{Tr}\left(\mathbf{A}_{1} \boldsymbol{\Sigma}_{i j}\right)\right] \\
& =\left(\boldsymbol{\mu}_{i j}-\boldsymbol{\mu}_{e}\right)^{\mathrm{T}} \mathbf{A}_{1}\left(\boldsymbol{\mu}_{i j}-\boldsymbol{\mu}_{e}\right)+\operatorname{Tr}\left(\mathbf{A}_{1} \boldsymbol{\Sigma}_{i j}\right)+\operatorname{Tr}\left(\mathbf{A}_{1} \boldsymbol{\Sigma}_{e}\right) \\
& =\left(\mu_{i j}^{(1)}-\mu_{e}^{(1)}\right)^{2}+\left(\boldsymbol{\Sigma}_{i j}\right)_{1,1}+\left(\boldsymbol{\Sigma}_{e}\right)_{1,1}
\end{aligned}
$$

with $\mu_{i j}^{(1)}$ denoting the first (i.e., horizontal) component of the mean flow vector at pixel $(i, j)$. The term $g_{\mathrm{C}}$ in $(5)$ can be determined as

$$
\begin{aligned}
g_{\mathrm{C}}\left(\boldsymbol{\mu}_{i j}, \boldsymbol{\Sigma}_{i j}, \hat{\boldsymbol{\mu}}_{i j}, \hat{\boldsymbol{\Sigma}}_{i j}\right) & =\mathbb{E}_{q(\mathbf{y}, \hat{\mathbf{y}} ; \boldsymbol{\theta})} f_{\mathrm{C}}\left(\mathbf{y}_{i j}, \hat{\mathbf{y}}_{i j}\right)^{2} \\
& =\mathbb{E}_{q\left(\mathbf{y}_{i j} ; \boldsymbol{\theta}\right)} \mathbb{E}_{q\left(\hat{\mathbf{y}}_{i j} ; \boldsymbol{\theta}\right)}\left[\left(\mathbf{y}_{i j}-\hat{\mathbf{y}}_{i j}\right)^{\mathrm{T}}\left(\mathbf{y}_{i j}-\hat{\mathbf{y}}_{i j}\right)\right] \\
& =\mathbb{E}_{q\left(\hat{\mathbf{y}}_{i j} ; \boldsymbol{\theta}\right)}\left[\left(\boldsymbol{\mu}_{i j}-\hat{\mathbf{y}}_{i j}\right)^{\mathrm{T}}\left(\boldsymbol{\mu}_{i j}-\hat{\mathbf{y}}_{i j}\right)+\operatorname{Tr}\left(\boldsymbol{\Sigma}_{i j}\right)\right] \\
& =\left(\boldsymbol{\mu}_{i j}-\hat{\boldsymbol{\mu}}_{i j}\right)^{\mathrm{T}}\left(\boldsymbol{\mu}_{i j}-\hat{\boldsymbol{\mu}}_{i j}\right)+\operatorname{Tr}\left(\boldsymbol{\Sigma}_{i j}\right)+\operatorname{Tr}\left(\hat{\boldsymbol{\Sigma}}_{i j}\right) .
\end{aligned}
$$

Update equations. To obtain update equations, we compute the derivative of the KL divergence in Eq. 22b], set it to zero, and solve for the desired variable. Please note that update equations for boundary pixels may slightly differ from the ones shown below. From now on, spatial derivatives of $I$ are denoted as $I_{x}$ and $I_{y}$, the temporal derivative is given as $I_{t}$. Moreover, $\operatorname{diag}(\cdot)$ represents a diagonal matrix and we define vectors $\mathbf{K}_{\gamma}=\left(\sum_{l} \frac{\mathbf{k}_{\gamma, i j, l}}{\sigma_{l}^{2}}\right)_{i j}$.

As the update of each mean flow estimate $\boldsymbol{\mu}_{i j}$ depends on other entries of $\boldsymbol{\mu}$, it is desirable to jointly solve for all components of the flow field. Therefore, $\boldsymbol{\mu}$ is obtained as the solution of a linear equation system, c.f. [50, 37], such that

$$
\mathbf{A} \mathbf{x}=\mathbf{b}, \quad \mathbf{x}=\left(\mu_{11}^{(1)}, \ldots, \mu_{n m}^{(1)}, \mu_{11}^{(2)}, \ldots, \mu_{n m}^{(2)}\right)^{\mathrm{T}}, \quad \mathbf{A}=\mathbf{A}_{\mathrm{D}}+\mathbf{A}_{\mathrm{S}}+\mathbf{A}_{\mathrm{C}}, \quad \mathbf{b}=\mathbf{b}_{\mathrm{D}}+\mathbf{b}_{\mathrm{S}}+\mathbf{b}_{\mathrm{C}}
$$

The components of the linear equation system are determined as

$$
\begin{aligned}
& \mathbf{A}_{\mathrm{D}}=\lambda_{\mathrm{D}}\left(\begin{array}{cc}
\operatorname{diag}\left(\mathbf{K}_{\mathrm{D}}\right) \operatorname{diag}\left(I_{x}^{2}\right) & \operatorname{diag}\left(\mathbf{K}_{\mathrm{D}}\right) \operatorname{diag}\left(I_{x} \cdot I_{y}\right) \\
\operatorname{diag}\left(\mathbf{K}_{\mathrm{D}}\right) \operatorname{diag}\left(I_{x} \cdot I_{y}\right) & \operatorname{diag}\left(\mathbf{K}_{\mathrm{D}}\right) \operatorname{diag}\left(I_{y}^{2}\right)
\end{array}\right), \\
& \mathbf{A}_{\mathrm{S}}=\lambda_{\mathrm{S}}\left(\begin{array}{cc}
\mathbf{F}_{1}^{\mathrm{T}} \operatorname{diag}\left(\mathbf{K}_{\mathrm{S}_{1}}\right) \mathbf{F}_{1}+\mathbf{F}_{2}^{\mathrm{T}} \operatorname{diag}\left(\mathbf{K}_{\mathrm{S}_{2}}\right) \mathbf{F}_{2} & \mathbf{0} \\
\mathbf{0} & \mathbf{F}_{1}^{\mathrm{T}} \operatorname{diag}\left(\mathbf{K}_{\mathrm{S}_{3}}\right) \mathbf{F}_{1}+\mathbf{F}_{2}^{\mathrm{T}} \operatorname{diag}\left(\mathbf{K}_{\mathrm{S}_{4}}\right) \mathbf{F}_{2}
\end{array}\right), \\
& \mathbf{A}_{\mathrm{C}}=2 \lambda_{\mathrm{C}} \mathbf{I}, \\
& \mathbf{b}_{\mathrm{D}}=\mathbf{A}_{D} \mathbf{y}_{0}-\lambda_{\mathrm{D}}\left(\begin{array}{l}
\operatorname{diag}\left(\mathbf{K}_{\mathrm{D}}\right) \operatorname{diag}\left(I_{x} \cdot I_{t}\right) \mathbf{1} \\
\operatorname{diag}\left(\mathbf{K}_{\mathrm{D}}\right) \operatorname{diag}\left(I_{y} \cdot I_{t}\right) \mathbf{1}
\end{array}\right), \mathbf{b}_{\mathrm{S}}=\mathbf{0}, \mathbf{b}_{\mathrm{C}}=2 \lambda_{\mathrm{C}} \hat{\boldsymbol{\mu}} .
\end{aligned}
$$


Here, $\mathbf{F}_{1}$ and $\mathbf{F}_{2}$ represent filter matrices corresponding to the derivative filters $\mathbf{H}_{1}=[1,-1]^{\mathrm{T}}$ and $\mathbf{H}_{2}=[1,-1]$, which are used in $f_{\mathrm{S}_{e}}\left(\mathbf{y}_{i j}, \mathbf{y}_{e}\right)$, c.f. [37]. $\mathbf{I}$ is the identity matrix and $\mathbf{0}$ is a matrix of all zeros.

When updating the auxiliary flow means $\hat{\boldsymbol{\mu}}$, a $5 \times 5$ neighborhood has to be considered. Therefore, a joint update of all estimates is computationally expensive and we follow [43] assuming fixed values for neighboring pixels, i.e.

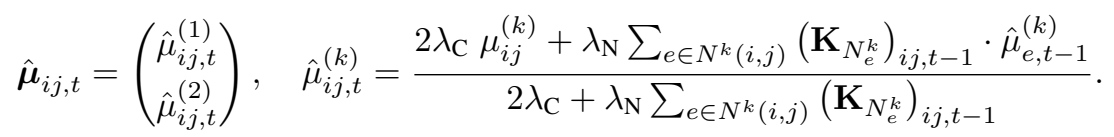

Here, $N^{k}(i, j)$ represents the set of neighbors in terms of the $k^{\text {th }}$ optical flow component.

For the flow variances $\Sigma=\left(\begin{array}{cc}\Sigma_{1} & 0 \\ 0 & \Sigma_{2}\end{array}\right)$ we derive a closed-form update dependent only on the latent variables $\mathbf{k}$ with

$$
\begin{aligned}
\Sigma_{1} & =\left(\lambda_{\mathrm{D}} \operatorname{diag}\left(I_{x}^{2}\right) \cdot \mathbf{K}_{\mathrm{D}}+\lambda_{\mathrm{S}}\left[\operatorname{abs}\left(\mathbf{F}_{1}^{\mathrm{T}}\right) \cdot \mathbf{K}_{\mathrm{S}_{1}}+\operatorname{abs}\left(\mathbf{F}_{2}^{\mathrm{T}}\right) \cdot \mathbf{K}_{\mathrm{S}_{2}}\right]+2 \lambda_{\mathrm{C}}\right)^{-1} \\
\text { and } \quad & \Sigma_{2}=\left(\lambda_{\mathrm{D}} \operatorname{diag}\left(I_{y}^{2}\right) \cdot \mathbf{K}_{\mathrm{D}}+\lambda_{\mathrm{S}}\left[\operatorname{abs}\left(\mathbf{F}_{1}^{\mathrm{T}}\right) \cdot \mathbf{K}_{\mathrm{S}_{3}}+\operatorname{abs}\left(\mathbf{F}_{2}^{\mathrm{T}}\right) \cdot \mathbf{K}_{\mathrm{S}_{4}}\right]+2 \lambda_{\mathrm{C}}\right)^{-1},
\end{aligned}
$$

where the absolute value function $\operatorname{abs}(\cdot)$ is applied element-wise.

We assume fixed neighboring values also for the update of the auxiliary flow variances $\hat{\boldsymbol{\Sigma}}$, and obtain

$$
\hat{\boldsymbol{\Sigma}}_{i j, t}=\left(\begin{array}{cc}
\hat{\Sigma}_{i j, t}^{(1)} & 0 \\
0 & \hat{\Sigma}_{i j, t}^{(2)}
\end{array}\right), \quad \hat{\Sigma}_{i j, t}^{(k)}=\frac{1}{2 \lambda_{\mathrm{C}}+\lambda_{\mathrm{N}} \sum_{e \in N^{k}(i, j)}\left(\mathbf{K}_{N_{e}^{k}}\right)_{i j, t-1}} .
$$

To derive an update equation for a latent variable $\mathbf{k}_{\gamma, i j}$, we need to consider a Lagrangian function including the KL divergence in Eq. 22b] as well as the constraint $\sum_{l} k_{\gamma, i j, l}=1$. Solving the resulting linear equation system analytically gives us, e.g.,

$$
\begin{aligned}
k_{\mathrm{D}, i j, l} & =\left(\frac{\pi_{l}}{\sigma_{l}}\right)^{\lambda_{\mathrm{D}}} \exp \left[-\lambda_{\mathrm{D}} \frac{g_{\mathrm{D}}\left(\boldsymbol{\mu}_{i j}, \boldsymbol{\Sigma}_{i j} ; I\right)}{2 \sigma_{l}^{2}}\right] \cdot Z_{\mathrm{D}, i j} \\
\text { with } \quad Z_{\mathrm{D}, i j} & =\left(\sum_{l=1}^{L}\left(\frac{\pi_{l}}{\sigma_{l}}\right)^{\lambda_{\mathrm{D}}} \exp \left[-\lambda_{\mathrm{D}} \frac{g_{\mathrm{D}}\left(\boldsymbol{\mu}_{i j}, \boldsymbol{\Sigma}_{i j} ; I\right)}{2 \sigma_{l}^{2}}\right]\right)^{-1}
\end{aligned}
$$

for the latent variables of the data term using the expectation values $g_{\mathrm{D}}\left(\boldsymbol{\mu}_{i j}, \boldsymbol{\Sigma}_{i j} ; I\right)$ as derived in Eq. 28d. Update equations for the remaining latent variables are derived similarly.

\section{B. Bayesian Risk Minimization}

We aim to show that the solution of the Bayesian risk minimization in Eq. 8) is given as $\mathbf{y}_{i j}^{\star}=\boldsymbol{\mu}_{i j}$ when replacing the posterior $p$ with its approximating distribution $q$ and using the Average End-Point Error (AEPE) as a loss function.

Recall that the AEPE is defined as $l(\mathbf{y}, \tilde{\mathbf{y}})=\sum_{i, j} \ell\left(\mathbf{y}_{i j}, \tilde{\mathbf{y}}_{i j}\right)=\sum_{i, j}\left\|\mathbf{y}_{i j}-\tilde{\mathbf{y}}_{i j}\right\|_{2}$ with

$$
\begin{aligned}
\nabla_{2} \ell(\mathbf{a}-\mathbf{x}, \tilde{\mathbf{x}}) & =(\mathbf{a}-\mathbf{x}-\tilde{\mathbf{x}}) /\|\mathbf{a}-\mathbf{x}-\tilde{\mathbf{x}}\|_{2} \\
& =-(\mathbf{x}-(\mathbf{a}-\tilde{\mathbf{x}})) /\|\mathbf{x}-(\mathbf{a}-\tilde{\mathbf{x}})\|_{2} \\
& =-\nabla_{2} \ell(\mathbf{x}, \mathbf{a}-\tilde{\mathbf{x}})
\end{aligned}
$$

for arbitrary $\mathbf{a} \in \mathbb{R}^{2}$. W.l.o.g. we minimize the expected risk of $l(\mathbf{y}, \tilde{\mathbf{y}})$ and therefore set $f(\tilde{\mathbf{y}})=\mathbb{E}_{q(\mathbf{y}, \hat{\mathbf{y}}, \mathbf{h} ; \boldsymbol{\theta})}[l(\mathbf{y}, \tilde{\mathbf{y}})]$. Note that we omit the variational parameters $\boldsymbol{\theta}$ in the following for brevity. Using the properties of $q$, we obtain

$$
\begin{aligned}
f(\tilde{\mathbf{y}}) & =\int_{\mathcal{Y}} \int_{\hat{\mathcal{Y}}} \sum_{\mathcal{H}} q(\mathbf{y}, \hat{\mathbf{y}}, \mathbf{h}) \cdot l(\mathbf{y}, \tilde{\mathbf{y}}) d \mathbf{y} d \hat{\mathbf{y}} \\
\stackrel{\text { qfac. }}{=} \int_{\mathcal{Y}} q(\mathbf{y}) \cdot l(\mathbf{y}, \tilde{\mathbf{y}}) d \mathbf{y} & \\
& =\sum_{i, j} \underbrace{\int_{\mathbb{R}^{2}} q\left(\mathbf{y}_{i j}\right) \cdot \ell\left(\mathbf{y}_{i j}, \tilde{\mathbf{y}}_{i j}\right) d \mathbf{y}_{i j}}_{=: f_{i j}\left(\tilde{\mathbf{y}}_{i j}\right)} .
\end{aligned}
$$


For fixed $\mathbf{y}_{i j} \in \mathbb{R}^{2}$, the function $q\left(\mathbf{y}_{i j}\right) \cdot \ell\left(\mathbf{y}_{i j}, \tilde{\mathbf{y}}_{i j}\right)$ is convex in $\tilde{\mathbf{y}}_{i j}$. Therefore, the objective $f(\tilde{\mathbf{y}})$ is convex in $\tilde{\mathbf{y}}$ and the Bayesian risk minimization has a unique solution given by

$$
\mathbf{y}_{i j}=\underset{\tilde{\mathbf{y}}_{i j}}{\arg \min } f_{i j}\left(\tilde{\mathbf{y}}_{i j}\right)
$$

It only remains to be shown that $\nabla_{2} f_{i j}\left(\tilde{\mathbf{y}}_{i j}\right)=0$ holds for $\tilde{\mathbf{y}}_{i j}=\boldsymbol{\mu}_{i j}$. Setting $\tilde{\mathbf{y}}_{i j}=\boldsymbol{\mu}_{i j}$ we obtain

$$
\begin{aligned}
& \int_{-\infty}^{\boldsymbol{\mu}_{i j}} q(\boldsymbol{\tau}) \cdot \nabla_{2} \ell\left(\boldsymbol{\tau}, \boldsymbol{\mu}_{i j}\right) d \boldsymbol{\tau} \stackrel{\left(\mathbf{z}_{1}=\boldsymbol{\tau}-\boldsymbol{\mu}_{i j}\right)}{=} \int_{-\infty}^{\mathbf{0}} q\left(\boldsymbol{\mu}_{i j}+\mathbf{z}_{1}\right) \cdot \nabla_{2} \ell\left(\boldsymbol{\mu}_{i j}+\mathbf{z}_{1}, \boldsymbol{\mu}_{i j}\right) d \mathbf{z}_{1} \\
& \stackrel{q \text { sym. }}{=} \quad \int_{-\infty}^{\mathbf{0}} q\left(\boldsymbol{\mu}_{i j}-\mathbf{z}_{1}\right) \cdot \nabla_{2} \ell\left(\boldsymbol{\mu}_{i j}+\mathbf{z}_{1}, \boldsymbol{\mu}_{i j}\right) d \mathbf{z}_{1} \\
& \left(\mathbf{z}_{2}=\stackrel{\left.\boldsymbol{\mu}_{i j}-\mathbf{z}_{1}\right)}{=} \int_{\boldsymbol{\mu}_{i j}}^{\infty} q\left(\mathbf{z}_{2}\right) \cdot \nabla_{2} \ell\left(2 \boldsymbol{\mu}_{i j}-\mathbf{z}_{2}, \boldsymbol{\mu}_{i j}\right) d \mathbf{z}_{2}\right. \\
& 37 a=37 c-\int_{\boldsymbol{\mu}_{i j}}^{\infty} q\left(\mathbf{z}_{2}\right) \cdot \nabla_{2} \ell\left(\mathbf{z}_{2}, \boldsymbol{\mu}_{i j}\right) d \mathbf{z}_{2}
\end{aligned}
$$

and finally

$$
\begin{aligned}
\nabla_{2} f_{i j}\left(\boldsymbol{\mu}_{i j}\right) & =\int_{\mathbb{R}^{2}} q(\boldsymbol{\tau}) \cdot \nabla_{2} \ell\left(\boldsymbol{\tau}, \boldsymbol{\mu}_{i j}\right) d \boldsymbol{\tau} \\
& =\int_{-\infty}^{\boldsymbol{\mu}_{i j}} q(\boldsymbol{\tau}) \cdot \nabla_{2} \ell\left(\boldsymbol{\tau}, \boldsymbol{\mu}_{i j}\right) d \boldsymbol{\tau}+\int_{\boldsymbol{\mu}_{i j}}^{\infty} q(\boldsymbol{\tau}) \cdot \nabla_{2} \ell\left(\boldsymbol{\tau}, \boldsymbol{\mu}_{i j}\right) d \boldsymbol{\tau} \\
& \stackrel{40 d]}{=}-\int_{\boldsymbol{\mu}_{i j}}^{\infty} q(\boldsymbol{\tau}) \cdot \nabla_{2} \ell\left(\boldsymbol{\tau}, \boldsymbol{\mu}_{i j}\right) d \boldsymbol{\tau}+\int_{\boldsymbol{\mu}_{i j}}^{\infty} q(\boldsymbol{\tau}) \cdot \nabla_{2} \ell\left(\boldsymbol{\tau}, \boldsymbol{\mu}_{i j}\right) d \boldsymbol{\tau} \\
& =0 .
\end{aligned}
$$

\section{Implementation Details}

In this section, we present our design choices following the best-practices of energy-based optical flow techniques, and give an analysis evaluating the influence of the specifics. Moreover, we give details of our post-processing approach using the fast bilateral solver [4].

\section{C.1. ProbClassicA}

In our ProbClassicA algorithm, we perform three steps of graduated non-convexity and apply coarse-to-fine estimation with 10 warping steps per layer. As in [43], we restrict the flow update to an absolute value of 1 and pre-process the images using a structure-texture decomposition. Spline-based cubic interpolation as well as an averaging of image gradients $\nabla_{2} I_{1}$ and $\nabla_{2} I_{2}$ are applied. During the inference, the variable sets $\{\boldsymbol{\mu}, \boldsymbol{\Sigma}, \mathbf{k}\}$ and $\{\hat{\boldsymbol{\mu}}, \hat{\boldsymbol{\Sigma}}, \hat{\mathbf{k}}\}$ are updated in an alternating way. As an inner update step, we apply five iterations of the block-coordinate descent scheme on $\boldsymbol{\mu}, \boldsymbol{\Sigma}$ and $\mathbf{k}$. For the set $\{\hat{\boldsymbol{\mu}}, \hat{\boldsymbol{\Sigma}}, \hat{\mathbf{k}}\}$, a number of three inner updates performs better.

\section{C.2. ProbFlowFields}

For ProbFlowFields, we follow [36] and pre-smooth images using a Gaussian kernel of size $9 \times 9$ with $\sigma=1.1$. For warping, we apply bilinear interpolation and averaged image derivatives. Moreover, we perform five warping steps, each with five iterations of our block-coordinate descent scheme. We follow Revaud et al. and compute optical flow updates with 30 iterations of successive over relaxation, which performs noticeably faster than the solver used in [43].

\section{C.3. Evaluation of design choices}

Table 6 summarizes results of AEPE, AUC, and CC on the Middlebury and Sintel benchmarks using varying setups of ProbClassicA and ProbFlowFields. In a first step, we evaluate a setting for ProbClassicA in which parameters $\lambda_{\mathrm{D}}, \lambda_{\mathrm{S}}$, and $\lambda_{\mathrm{N}}$ are determined by having the Bayesian optimization [42] consider only the AEPE or only the AUC instead of the $F_{1}$-score 


\begin{tabular}{|c|c|c|c|c|c|c|}
\hline ProbClassicA Middlebury & AEPE & rel. chg. & AUC & rel. chg. & $\mathrm{CC}$ & rel. chg. \\
\hline Baseline & 0.296 & - & 0.466 & - & 0.374 & - \\
\hline Bayesian optim. w.r.t. AEPE only & 0.290 & -0.02 & 0.471 & 0.01 & 0.351 & 0.06 \\
\hline Bayesian optim. w.r.t. AUC only & 0.312 & 0.05 & 0.436 & -0.06 & 0.451 & -0.21 \\
\hline$E_{\mathrm{N}}=E_{\mathrm{C}}=0$ & 0.411 & 0.39 & 0.889 & 0.91 & 0.125 & 0.67 \\
\hline No structure-texture decomposition & 0.290 & -0.02 & 0.445 & -0.05 & 0.361 & 0.03 \\
\hline ProbFlowFields Sintel validation & AEPE & rel. chg. & AUC & rel. chg. & $\mathrm{CC}$ & rel. chg. \\
\hline Baseline & 3.127 & - & 0.398 & - & 0.563 & - \\
\hline Bayesian optim. w.r.t. AEPE only & 3.128 & $<0.01$ & 0.475 & 0.19 & 0.407 & 0.28 \\
\hline Bayesian optim. w.r.t. AUC only & 3.219 & $\mathbf{0 . 0 3}$ & 0.381 & -0.04 & 0.644 & -0.14 \\
\hline Spatially constant $\lambda_{\mathrm{S}}$ & 3.127 & 0.00 & 0.400 & $<0.01$ & 0.562 & $<0.01$ \\
\hline$\theta_{i j}^{r}=1$ & 3.125 & $>-0.01$ & 0.396 & $>-0.01$ & 0.548 & 0.03 \\
\hline No gradient averaging & 3.135 & $<0.01$ & 0.398 & 0.00 & 0.557 & 0.01 \\
\hline No Gaussian smoothing & 3.135 & $<0.01$ & 0.441 & 0.11 & 0.497 & 0.12 \\
\hline 10 warping steps & 3.123 & $>-0.01$ & 0.421 & 0.06 & 0.538 & 0.04 \\
\hline
\end{tabular}

Table 6. Analysis of several design choices for ProbClassicA on Middlebury and ProbFlowFields on the Sintel validation set. Bold entries denote strong deviations from the baseline.

proposed in Eq. 20. In both cases, we observe that the performance w.r.t. the evaluation metric that is not considered during the Bayesian optimization drops significantly. This highlights the importance of the $\mathrm{F}_{1}$-score to balance the accuracy of flow and uncertainty estimates. Moreover, we show that the AEPE as well as the performance of the uncertainty measure is clearly inferior if no additional nonlocal term is applied $\left(E_{\mathrm{N}}=E_{\mathrm{C}}=0\right)$. When using ProbClassicA without structure-texture decomposition as pre-processing, we surprisingly obtain improved results for the AEPE (2\%) as well as the AUC (5\%). This is in contrast to energy minimization, where this pre-processing helps [43]. For fairness of comparison to the underlying energy minimization approach, we continue to use a structure-texture decomposition.

Considering ProbFlowFields, we observe the same behavior as for ProbClassicA when Bayesian optimization is carried out only with respect to one of the evaluation metrics. Note that the parameter setting obtained by a Bayesian optimization w.r.t. to the AEPE performs better than the baseline on the training set even though no improvement of the AEPE is visible on the validation set. The usage of a spatially constant trade-off parameter $\lambda_{\mathrm{S}}$, turning off the normalization of the spatial derivatives $\left(\theta_{i j}^{r}=1, c . f\right.$. Eqs. (17) and (18)), and not averaging the image gradients, respectively, only lead to minor changes. When no Gaussian smoothing is applied for image pre-processing, a clear effect on the AUC as well as the CC can be observed whereas the AEPE is only slightly changed. Finally, the application of 10 warping steps only results in small improvements of the AEPE and even decreases the performance of the uncertainty measure. This justifies the usage of a reduced number of 5 steps to save computational time.

\section{C.4. Post-processing using the fast bilateral solver}

As described in Sec.7.3, we apply the fast bilateral solver [4] on top of ProbFlowFields in order to illustrate the benefits of uncertainty predictions for a further improvement of the flow estimates. In doing so, we normalize the estimated uncertainties with a sigmoid function and invert the values to obtain the confidences required by the fast bilateral solver. A Bayesian optimization [42] is performed on our Sintel training set to obtain appropriate sigmoid parameters as well as a suitable trade-off parameter for the fast bilateral solver. See Fig. 5f for a screenshot of the private Sintel benchmark table showing results after post-processing (ProbFlowFields + BS). For the reported baseline, we process the estimates of ProbFlowFields assuming a uniform confidence of 0.5 .

\section{Additional Uncertainty Measures}

In the following, we evaluate several additional uncertainty measures on the Middlebury as well as the Sintel benchmark. Haußecker and Spies [20] introduce three confidence measures based on the spatio-temporal structure tensor

$$
\mathbf{S}=G(\tilde{\sigma}) *\left[\left(\nabla_{3} I\right)\left(\nabla_{3} I\right)^{\mathrm{T}}\right] \quad \text { with } \quad \nabla_{3} I=\left(I_{x}, I_{y}, I_{t}\right)^{\mathrm{T}},
$$

where $I_{x}$ and $I_{y}$ denote the spatial image derivatives computed with central differences and $I_{t}$ is the temporal difference between $I_{1}$ and $I_{2}$. Following [30], we smooth the derivatives with a Gaussian filter $G(\tilde{\sigma})$ of size $7 \times 7$ and a standard 


\begin{tabular}{|c|c|c|c|c|c|c|c|c|c|c|}
\hline & EPE all & EPE matched & EPE unmatched & do-10 & d10-60 & d60-140 & so-10 & $s 10-40$ & s40+ & \\
\hline GroundTruth ${ }^{[1]}$ & 0.000 & 0.000 & 0.000 & 0.000 & 0.000 & 0.000 & 0.000 & 0.000 & 0.000 & Visualize Results \\
\hline DCFlow ${ }^{[2]}$ & 5.119 & 2.283 & 28.228 & 4.665 & 2.108 & 1.440 & 1.052 & 3.434 & 29.351 & Visualize Results \\
\hline FlowFieldsCNN ${ }^{[3]}$ & 5.363 & 2.303 & 30.313 & 4.718 & 2.020 & 1.399 & 1.032 & 3.065 & 32.422 & Visualize Results \\
\hline MR-Flow ${ }^{[4]}$ & 5.376 & 2.818 & 26.235 & 5.109 & 2.395 & 1.755 & 0.908 & 3.443 & 32.221 & Visualize Results \\
\hline FTFlow ${ }^{[5]}$ & 5.390 & 2.268 & 30.841 & 4.513 & 1.964 & 1.366 & 1.046 & 3.322 & 31.936 & Visualize Results \\
\hline S2F-IF ${ }^{[6]}$ & 5.417 & 2.549 & 28.795 & 4.745 & 2.198 & 1.712 & 1.157 & 3.468 & 31.262 & Visualize Results \\
\hline InterpoNet_ff [7] & 5.535 & 2.372 & 31.296 & 4.720 & 2.018 & 1.532 & 1.064 & 3.496 & 32.633 & Visualize Results \\
\hline RegionalFF ${ }^{[8]}$ & 5.562 & 2.595 & 29.741 & 4.921 & 2.393 & 1.639 & 1.122 & 3.477 & 32.625 & Visualize Results \\
\hline PGM-C ${ }^{[9]}$ & 5.591 & 2.672 & 29.389 & 4.975 & 2.340 & 1.791 & 1.057 & 3.421 & 33.339 & Visualize Results \\
\hline RicFlow ${ }^{[10]}$ & 5.620 & 2.765 & 28.907 & 5.146 & 2.366 & 1.679 & 1.088 & 3.364 & 33.573 & Visualize Results \\
\hline InterpoNet_cpm [11] & 5.627 & 2.594 & 30.344 & 4.975 & 2.213 & 1.640 & 1.042 & 3.575 & 33.321 & Visualize Results \\
\hline ProbFlowFields+BS ${ }^{[12]}$ & 5.628 & 2.543 & 30.773 & 4.680 & 2.169 & 1.683 & 1.086 & 3.538 & 33.210 & Visualize Results \\
\hline CPM_AUG ${ }^{[13]}$ & 5.645 & 2.737 & 29.362 & 4.707 & 2.150 & 1.918 & 1.087 & 3.306 & 33.925 & Visualize Results \\
\hline ProbFlowFields ${ }^{[14]}$ & 5.696 & 2.545 & 31.371 & 4.696 & 2.150 & 1.686 & 1.146 & 3.658 & 33.188 & Visualize Results \\
\hline
\end{tabular}

Figure 5. Screenshot of private Sintel table (final) showing results for ProbFlowFields and ProbFlowFields + BS (status as of July 2017).

\begin{tabular}{lcccc}
\hline Uncertainty measure & AUC & rel. chg. & CC & rel. chg. \\
\hline Ct [20] & 1.058 & 1.27 & -0.106 & 1.28 \\
Cs [20] & 1.014 & 1.18 & -0.057 & 1.15 \\
Cc [20] & 0.967 & 1.08 & -0.022 & 1.06 \\
Ev3 [27] & 0.989 & 1.12 & 0.058 & 0.84 \\
Noise & 0.512 & 0.10 & 0.286 & 0.24 \\
ProbClassicA (ours) & $\mathbf{0 . 4 6 6}$ & $\mathbf{0 . 0 0}$ & $\mathbf{0 . 3 7 4}$ & $\mathbf{0 . 0 0}$ \\
Oracle & 0.255 & - & 1.000 & - \\
\hline
\end{tabular}

Table 7. Area under curve (AUC), Spearman's rank correlation coefficient (CC), and relative change (rel. chg.) in comparison to the our uncertainty measure on the Middlebury dataset.

\begin{tabular}{lcccc}
\hline Uncertainty measure & AUC & rel. chg. & CC & rel. chg. \\
\hline Ct [20] & 1.130 & 1.84 & -0.128 & 1.23 \\
Cs [20] & 1.154 & 1.90 & -0.149 & 1.26 \\
Cc [20] & 0.915 & 1.30 & 0.129 & 0.77 \\
Ev3 [27] & 1.024 & 1.57 & -0.030 & 1.05 \\
Noise & 0.512 & 0.29 & 0.382 & 0.32 \\
ProbFlowFields (ours) & $\mathbf{0 . 3 9 8}$ & $\mathbf{0 . 0 0}$ & $\mathbf{0 . 5 6 3}$ & $\mathbf{0 . 0 0}$ \\
Oracle & 0.182 & - & 1.000 & - \\
\hline
\end{tabular}

Table 8. Area under curve (AUC), Spearman's rank correlation coefficient (CC), and relative change (rel. chg.) in comparison to our uncertainty measure on a Sintel benchmark validation set.

deviation $\tilde{\sigma}=2$. In [20], eigenvalues $\lambda_{1}, \lambda_{2}$, and $\lambda_{3}$ of $\mathbf{S}$ are computed such that $\lambda_{1} \geq \lambda_{2} \geq \lambda_{3}$. Uncertainty measures are then obtained as

$$
\Psi_{\mathrm{Ct}}=-\left(\frac{\lambda_{1}-\lambda_{3}}{\lambda_{1}+\lambda_{3}}\right)^{2}, \quad \Psi_{\mathrm{Cs}}=-\left(\frac{\lambda_{1}-\lambda_{2}}{\lambda_{1}+\lambda_{2}}\right)^{2}, \quad \text { and } \quad \Psi_{\mathrm{Cc}}=\Psi_{\mathrm{Ct}}-\Psi_{\mathrm{Cs}} .
$$

Moreover, we evaluate a baseline uncertainty measure as used in [27] defined as $\Psi_{\mathrm{Ev} 3}=-\lambda_{3}$.

Finally, we compare to a sampling-based measure similar to the idea of Kybic and Nieuwenhuis [27]. That is, we estimate the uncertainty as the variance of the optical flow estimates resulting from small, random perturbations of the input data. Specifically, we apply zero-mean Gaussian noise on the input images and determine appropriate values for the variance of the noise on the training set. The uncertainty measure is then obtained as $\Psi_{\text {Noise }}=\sqrt{\sigma_{u}^{2}+\sigma_{v}^{2}}$ with $\sigma_{u}$ and $\sigma_{v}$ denoting the standard derivation of the horizontal and vertical flow estimates per pixel.

As can be seen in Tables 7 and 8 , all measures based on the structure tensor perform considerably worse than our proposed uncertainty measure. $\Psi_{\mathrm{Cc}}$ and $\Psi_{\mathrm{Ev} 3}$ lead to more meaningful uncertainties than the two remaining approaches on both datasets, but perform similar to the simple gradient-based measure [3]. The noise uncertainty - especially on the Middlebury dataset - performs comparably to $\Psi_{\text {Energy }}$ and $\Psi_{\text {Learned. }}$. However, our ProbFlow approach clearly leads to superior results.

\section{E. ProbFlowFields on Middlebury}

For completeness, we report the results of ProbFlowFields on Middlebury. To reproduce the Middlebury results shown in [1] we applied the default settings of the EpicFlow interpolation. Moreover, we use GSM potentials trained on the Sintel 


\begin{tabular}{lccccc}
\hline & \multicolumn{2}{c}{ training } & & \multicolumn{2}{c}{ test } \\
\cline { 2 - 3 } \cline { 5 - 6 } Method & AEPE & rel. chg. & & AEPE & rel. chg. \\
\hline Initialization & 0.307 & 0.38 & & - & - \\
FlowFields [1] & 0.240 & 0.08 & & $0.331^{\dagger}$ & 0.10 \\
FieldsFields & 0.230 & 0.04 & & - & - \\
ProbFlowFields (ours) & $\mathbf{0 . 2 2 2}$ & $\mathbf{0 . 0 0}$ & & $\mathbf{0 . 3 0 1}$ & $\mathbf{0 . 0 0}$ \\
\hline
\end{tabular}

Table 9. Average end-point error (AEPE) and relative change (rel. chg.) in comparison to the ProbFlowFields method on the Middlebury benchmark. ${ }^{\dagger}$ Please note that we did not re-evaluate FlowFields, but show the publicly available results.

\begin{tabular}{lcccc}
\hline Uncertainty measure & AUC & rel. chg. & CC & rel. chg. \\
\hline Gradient [3] & 1.244 & 1.72 & -0.077 & 1.21 \\
Laplace & 0.539 & 0.18 & 0.297 & 0.20 \\
Energy [8] & 0.563 & 0.23 & 0.253 & 0.32 \\
Learned [30] & 0.473 & 0.04 & $\mathbf{0 . 3 7 4}$ & $>\mathbf{- 0 . 0 1}$ \\
ProbFlowFields (ours) & $\mathbf{0 . 4 5 7}$ & $\mathbf{0 . 0 0}$ & 0.371 & 0.00 \\
Oracle & 0.247 & - & 1.000 & - \\
\hline
\end{tabular}

Table 10. Area under curve (AUC), Spearman's rank correlation coefficient (CC), and relative change (rel. chg.) in comparison to the energy uncertainty measure on the Middlebury training set.

\begin{tabular}{|c|c|c|c|c|c|c|c|c|c|c|c|c|c|c|c|c|c|c|c|c|c|c|c|c|c|}
\hline \multirow[t]{2}{*}{$\begin{array}{c}\text { Average } \\
\text { endpoint } \\
\text { error }\end{array}$} & \multirow{2}{*}{$\begin{array}{l}\text { avg. } \\
\text { rank }\end{array}$} & \multicolumn{3}{|c|}{$\begin{array}{c}\text { Army } \\
\text { (Hidden texture) } \\
\text { GT im0 im1 }\end{array}$} & \multicolumn{3}{|c|}{$\begin{array}{c}\text { Mequon } \\
\text { (Hidden texture) } \\
\text { GT im0 im1 }\end{array}$} & \multicolumn{3}{|c|}{$\begin{array}{c}\text { Schefflera } \\
\text { (Hidden texture) } \\
\text { GT im0 im1 }\end{array}$} & \multicolumn{3}{|c|}{$\begin{array}{c}\text { Wooden } \\
\text { (Hidden texture) } \\
\text { GT im0 im1 }\end{array}$} & \multicolumn{3}{|c|}{$\begin{array}{c}\text { Grove } \\
\text { (Synthetic) } \\
\text { GT im0 im1 }\end{array}$} & \multicolumn{3}{|c|}{$\begin{array}{c}\text { Urban } \\
\text { (Synthetic) } \\
\text { GI im0 im1 }\end{array}$} & \multicolumn{3}{|c|}{$\begin{array}{c}\text { Yosemite } \\
\text { (Synthetic) } \\
\text { GT im0 im1 }\end{array}$} & \multicolumn{3}{|c|}{$\begin{array}{c}\text { Teddy } \\
\text { (Stereo) } \\
\text { GT } \stackrel{\text { im0 }}{i m 1}\end{array}$} \\
\hline & & all & disc & untext & all & disc & untext & all & & untext & all & & untext & all & disc & untext & all & & untext & all & & untext & & & untext \\
\hline NNF-Local [87] & 3.4 & $\underline{0.07} 1$ & $\overline{0.202}$ & $\overline{0.051}$ & $\underline{0.152}$ & $\overline{0.514}$ & $\overline{0.125}$ & $\underline{0.182}$ & 0.372 & $\overline{0.142}$ & $\underline{0.102} 2$ & $\overline{0.495}$ & $\overline{0.062}$ & $\underline{0.41} 1$ & $\overline{0.61} 1$ & $\overline{0.212}$ & $\underline{0.232}$ & $0.66_{3}$ & $\overline{0.192}$ & $\underline{0.106} 6$ & 0.1212 & 0.1713 & 0.342 & $0.80_{6}$ & 0.232 \\
\hline PMMST [114] & 9.3 & $\underline{0.09} 32$ & 0.215 & 0.0716 & 0.1811 & 0.514 & 0.1628 & $\underline{0.219}$ & 0.428 & 0.1716 & $\underline{0.10} 2$ & 0.331 & 0.0813 & $\underline{0.515}$ & 0.744 & 0.287 & $\underline{0.243}$ & 0.652 & 0.205 & 0.1118 & 0.1212 & 0.1713 & $\underline{0.374}$ & 0.742 & 0.354 \\
\hline OFLAF [77] & 9.8 & 0.089 & 0.215 & 0.066 & 0.166 & 0.536 & 0.125 & $\underline{0.19} 3$ & 0.372 & 0.142 & 0.149 & 0.7729 & 0.075 & 0.515 & 0.787 & 0.254 & 0.3111 & 0.764 & 0.2516 & 0.1118 & 0.1212 & 0.2140 & 0.4210 & 0.784 & 0.6318 \\
\hline MDP-Flow2 [68] & 10.6 & $\underline{0.089} 9$ & 0.215 & 0.0716 & $\underline{0.15} 2$ & $0.48_{1}$ & 0.111 & $\underline{0.20} 5$ & 0.405 & 0.142 & 0.1522 & 0.8037 & 0.0813 & 0.6319 & 0.9319 & 0.4320 & 0.265 & 0.764 & 0.239 & 0.1118 & 0.1212 & 0.1713 & 0.386 & 0.795 & 0.446 \\
\hline NN-field [71] & 11.8 & 0.089 & 0.2217 & 0.051 & 0.178 & 0.5510 & 0.1311 & $\underline{0.19} 3$ & 0.394 & 0.157 & 0.091 & 0.484 & 0.051 & 0.411 & 0.611 & 0.201 & 0.5260 & 0.641 & 0.2619 & 0.1342 & 0.1336 & 0.2034 & $\underline{0.35} 3$ & 0.838 & 0.211 \\
\hline ComponentFusion [96] & 13.9 & 0.071 & 0.215 & 0.051 & $\underline{0.16} 6$ & 0.5510 & 0.125 & $\underline{0.20} 5$ & 0.449 & 0.157 & $\underline{0.114}$ & 0.659 & 0.062 & $\underline{0.71} 35$ & 1.0740 & 0.5337 & 0.3215 & 1.0627 & 0.2822 & 0.1118 & 0.1336 & 0.158 & $\underline{0.419}$ & 0.8813 & 0.549 \\
\hline TC/T-Flow [76] & 19.5 & 0.07 & 0.215 & 0.051 & $\underline{0.19} 18$ & 0.6834 & 0.125 & 0.2826 & 0.6631 & 0.142 & $\underline{0.149}$ & 0.8647 & 0.075 & $\underline{0.67} 29$ & 0.9829 & 0.4931 & 0.22 & 0.829 & 0.192 & 0.1118 & 0.112 & 0.3085 & $\underline{0.50} 27$ & 1.0230 & 0.6420 \\
\hline WLIF-Flow [93] & 19.8 & 0.089 & 0.215 & 0.066 & 0.1811 & 0.5510 & 0.1523 & 0.2518 & 0.5620 & 0.1716 & 0.149 & 0.6810 & 0.0813 & 0.6116 & 0.9117 & 0.4118 & 0.4335 & 0.9615 & 0.2928 & 0.1342 & 0.1212 & 0.2140 & 0.5132 & 1.0333 & 0.7238 \\
\hline NNF-EAC [103] & 21.3 & 0.0932 & 0.2217 & 0.0716 & 0.178 & 0.536 & 0.1311 & 0.2311 & 0.4912 & 0.157 & 0.1636 & 0.8037 & 0.0928 & 0.6013 & 0.8913 & 0.4016 & 0.3825 & 0.786 & 0.2822 & 0.1231 & 0.1212 & 0.1826 & 0.5745 & 1.2449 & 0.6932 \\
\hline Layers++ [37] & 21.9 & 0.089 & 0.215 & 0.0716 & $\underline{0.19} 18$ & 0.5613 & 0.1735 & 0.205 & 0.405 & 0.1827 & 0.138 & 0.587 & 0.075 & 0.483 & 0.703 & 0.339 & 0.4747 & 1.0119 & 0.3347 & 0.1565 & 0.1458 & 0.2453 & $\underline{0.46} 17$ & 0.8813 & 0.7238 \\
\hline LME [70] & 22.9 & 0.089 & 0.2217 & 0.066 & 0.152 & 0.492 & 0.111 & 0.3035 & 0.6426 & 0.3189 & 0.1522 & 0.7833 & 0.0928 & 0.6625 & 0.9624 & 0.5337 & 0.3316 & 1.1844 & 0.2822 & 0.1231 & 0.1212 & 0.1826 & 0.4412 & 0.9116 & 0.6114 \\
\hline IROF++ [58] & 23.0 & $\underline{0.08} 9$ & 0.2324 & 0.0716 & $\underline{0.21} 32$ & 0.6834 & 0.1735 & 0.2826 & 0.6324 & 0.1939 & 0.1522 & 0.7322 & 0.0928 & 0.6013 & 0.8913 & 0.4219 & 0.4335 & 1.0830 & 0.3137 & 0.106 & 0.1212 & 0.124 & $\underline{0.47} 19$ & 0.9823 & 0.6831 \\
\hline nLayers [57] & 23.8 & 0.07 & 0.191 & 0.066 & 0.2240 & 0.5916 & 0.1957 & 0.2518 & 0.5416 & 0.2048 & 0.1522 & 0.8443 & 0.0813 & 0.537 & 0.787 & 0.3411 & 0.4439 & 0.8410 & 0.3033 & 0.1342 & 0.1336 & 0.2034 & 0.47 ig & 0.9722 & 0.6729 \\
\hline HAST [109] & & 0.07 & $0.20_{2}$ & 0.051 & 0.1811 & 0.548 & 0.1311 & 0.171 & 0.321 & 0.121 & 0.1522 & 0.9058 & 0.062 & 0.494 & 0.744 & 0.223 & 0.5870 & 1.0931 & 0.4470 & 0.1996 & 0.1787 & 0.47114 & $\underline{0.32}$ & 0.641 & \\
\hline PH-Flow [101] & 25.8 & 0.089 & 0.2431 & 0.0716 & $\underline{0.21} 32$ & 0.6834 & 0.1735 & 0.2311 & 0.4912 & 0.1939 & 0.1636 & 0.8341 & 0.0928 & $\underline{0.569} 9$ & 0.839 & 0.3813 & 0.309 & 0.817 & 0.2414 & 0.1565 & 0.1336 & 0.3085 & 0.4311 & 0.859 & 0.6627 \\
\hline FC-2Layers-FF [74] & & 0.089 & 0.215 & 0.0716 & 0.2132 & $0.70_{42}$ & 0.1735 & $\underline{0.20} 5$ & 0.405 & 0.1827 & 0.1522 & 0.7628 & 0.0813 & 0.537 & 0.776 & 0.3712 & 0.4953 & 1.0220 & 0.3347 & 0.1676 & 0.1336 & 0.2980 & 0.4412 & 0.8712 & 0.6420 \\
\hline Correlation Flow [75] & 26.5 & 0.0932 & 0.2324 & 0.0716 & 0.178 & 0.5815 & 0.111 & 0.4365 & 0.9967 & 0.157 & 0.114 & 0.473 & 0.0813 & 0.7541 & 1.0841 & 0.5642 & 0.4131 & 0.9213 & 0.3033 & 0.1452 & 0.1336 & 0.2769 & 0.408 & 0.859 & 0.425 \\
\hline & 28.0 & $\underline{0.08} 9$ & 0.2217 & 0.0716 & $\underline{0.23} 55$ & 0.7347 & 0.1847 & 0.2826 & 0.6631 & 0.1827 & 0.149 & 0.7013 & 0.0813 & 0.5710 & 0.8510 & 0.3813 & 0.4747 & 0.9716 & 0.3137 & 0.1342 & 0.1336 & 0.2246 & $\underline{0.51} 32$ & 0.9926 & 0.7447 \\
\hline RNLOD-Flow [121] & 28.0 & $\underline{0.07}$ & $0.20_{2}$ & 0.066 & $\underline{0.19} 18$ & 0.6834 & 0.1311 & 3349 & 0.7950 & 0.1716 & 0.149 & 0.7322 & 0.075 & 0.6933 & 1.0333 & 0.4827 & 0.3724 & 0.9917 & 0.2928 & 0.1676 & 0.1679 & 0.2980 & $\underline{0.45} 14$ & 0.8813 & 0.6525 \\
\hline ProbFlowFields [128] & 28.8 & $\underline{0.1047}$ & 0.3173 & 0.0844 & $\underline{0.19} 18$ & 0.6323 & 0.1735 & 0.2722 & 0.6324 & 0.2259 & 0.114 & 0.495 & 0.075 & 0.8251 & 1.2256 & 0.5945 & 0.254 & 1.0526 & 0.216 & 0.094 & 0.1212 & 0.1713 & $\underline{0.58} 47$ & 1.3351 & 0.6216 \\
\hline • & & & & & & & & & & & & & & & & & & & & & & & & 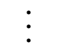 & \\
\hline & & & 0.3173 & 0.1191 & 0.2881 & & 0.2282 & & 1.0068 & & $\underline{0.2686}$ & & & & 1.2153 & & & & & & 0.112 & 0.2 & 9391 & 1.82 & \\
\hline ProbClassicA [127] & 66.5 & $\underline{0.10} 47$ & 0.2854 & 0.0844 & 0.2240 & 0.7861 & 0.1628 & 0.5788 & 1.1784 & 0.2259 & 0.2172 & 1.2486 & 0.1156 & 0.8658 & 1.3072 & 0.6352 & 0.5565 & 1.7493 & 0.3 & 0.1676 & 0.1458 & 0.3499 & 0.8182 & 1.7485 & 0.9371 \\
\hline TriangleFlow [30] & 66.7 & $\underline{0.11} 59$ & 0.2962 & 0.0965 & 0.2673 & 0.9588 & 0.1735 & 0.4774 & 1.0772 & 0.1827 & 0.1636 & 0.8751 & 0.0928 & 1.0797 & 1.47104 & 1.1098 & 0.8786 & 1.3965 & 0.5787 & 0.1565 & 0.19109 & 0.2352 & 0.6355 & 1.3351 & 0.8461 \\
\hline
\end{tabular}

Figure 6. Screenshot of private Middlebury table showing results for ProbFlowFields and ProbClassicA (status as of July 2017).

dataset for our ProbFlowFields approach. The results evaluating the AEPE on the Middlebury benchmark can be found in Table 9. We outperform the original FlowFields approach on training and test and obtain improved results in comparison to FlowFields ${ }^{\star}$. Please note that the Middlebury benchmark policy allows no more than one entry per method in the public table. Therefore, we decided to show the results of ProbFlowFields on the Middlebury website whereas the results of ProbClassicA from Table 1 of the main paper are only visible in a private table, see Fig. 6 for a screenshot.

Table 10 shows an evaluation of different uncertainty measures. In contrast to our remaining experiments, the Laplace and learned uncertainty measures both outperform the energy-based approach. Our uncertainty measure is slightly outperformed by $\Psi_{\text {Learned }}$ w.r.t. the CC metric. However, ProbFlowFields shows clearly superior results considering the AUC.

\section{References}

[50] T. Brox, A. Bruhn, N. Papenberg, and J. Weickert. High accuracy optical flow estimation based on a theory for warping. ECCV, 2004. 3 